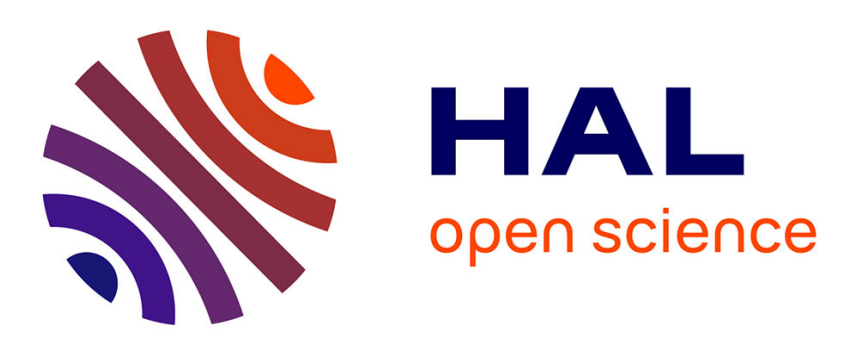

\title{
Using underdominance to bi-stably transform local populations
}

\author{
Philipp M. Altrock, Arne Traulsen, R. Guy Reeves, Floyd A. Reed
}

\section{To cite this version:}

Philipp M. Altrock, Arne Traulsen, R. Guy Reeves, Floyd A. Reed. Using underdominance to bi-stably transform local populations. Journal of Theoretical Biology, 2010, 267 (1), pp.62. 10.1016/j.jtbi.2010.08.004 . hal-00627145

\section{HAL Id: hal-00627145 https://hal.science/hal-00627145}

Submitted on 28 Sep 2011

HAL is a multi-disciplinary open access archive for the deposit and dissemination of scientific research documents, whether they are published or not. The documents may come from teaching and research institutions in France or abroad, or from public or private research centers.
L'archive ouverte pluridisciplinaire HAL, est destinée au dépôt et à la diffusion de documents scientifiques de niveau recherche, publiés ou non, émanant des établissements d'enseignement et de recherche français ou étrangers, des laboratoires publics ou privés. 


\section{Author's Accepted Manuscript}

Using underdominance to bi-stably transform local populations

Philipp M. Altrock, Arne Traulsen, R. Guy Reeves, Floyd A. Reed

$\begin{array}{ll}\text { PII: } & \text { S0022-5193(10)00403-0 } \\ \text { DOI: } & \text { doi:10.1016/j.jtbi.2010.08.004 } \\ \text { Reference: } & \text { YJTBI6108 }\end{array}$

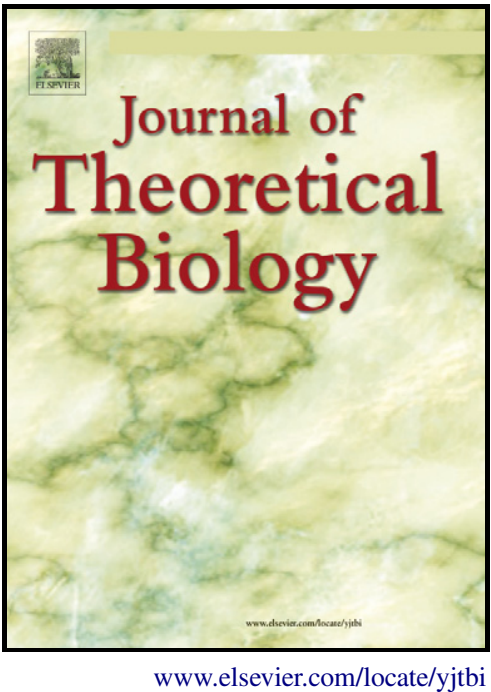

To appear in: $\quad$ Journal of Theoretical Biology

Received date: 2 February 2010

Revised date: $\quad 2$ August 2010

Accepted date: $\quad 2$ August 2010

Cite this article as: Philipp M. Altrock, Arne Traulsen, R. Guy Reeves and Floyd A. Reed, Using underdominance to bi-stably transform local populations, Journal of Theoretical Biology, doi:10.1016/j.jtbi.2010.08.004

This is a PDF file of an unedited manuscript that has been accepted for publication. As a service to our customers we are providing this early version of the manuscript. The manuscript will undergo copyediting, typesetting, and review of the resulting galley proof before it is published in its final citable form. Please note that during the production process errors may be discovered which could affect the content, and all legal disclaimers that apply to the journal pertain. 


\title{
Using underdominance to bi-stably transform local populations
}

\author{
Philipp M. Altrock ${ }^{a *}$, Arne Traulsen ${ }^{a \dagger}$, R. Guy Reeves ${ }^{b \ddagger}$, and Floyd A. Reed ${ }^{b \star}$ \\ a Research Group for Evolutionary Theory, \\ $b$ Population Genetics Group, Department of Evolutionary Genetics, \\ Max-Planck-Institute for Evolutionary Biology, \\ August-Thienemann-Str. 2, \\ D-24306 Plön, Germany \\ *altrock@evolbio.mpg.de, $\uparrow$ traulsen@evolbio.mpg.de, $\ddagger$ reeves@evolbio.mpg.de \\ $\star$ Corresponding author, reed@evolbio.mpg.de, Tel.: ++49(0)4522 763235 Fax: ++49(0)4522 763281
}

\begin{abstract}
Underdominance refers to natural selection against individuals with a heterozygous genotype. Here, we analyse a single-locus underdominant system of two large local populations that exchange individuals at a certain migration rate. The system can be characterized by fixed points in the joint allele frequency space. We address the conditions under which underdominance can be applied to transform a local population that is receiving wildtype immigrants from another population. In a single population, underdominance has the benefit of complete removal of genetically modified alleles (reversibility) and coexistence is not stable. The two population system that exchanges migrants can result in internal stable states, where coexistence is maintained, but with additional release of wildtype individuals the system can be reversed to a fully wildtype state. This property is critically controlled by the migration rate. We approximate the critical minimum frequency required to result in a stable population transformation. We also concentrate on the destabilizing effects of fitness and migration rate asymmetry. Practical implications of our results are discussed in the context of utilizing underdominance to genetically modify wild populations. This is of importance especially for genetic pest management strategies, where locally stable and potentially reversible transformations of populations of disease vector species are of interest.
\end{abstract}




\section{Introduction}

\subsection{Overview}

In population genetics, underdominance, also known as heterozygote disadvantage or homozygote advantage, refers to the fitness configuration where diploid individuals with heterozygote genotypes have a lower fitness than the corresponding homozygous genotypes. It is thus the opposite of overdominance or heterozygote advantage. Here lower fitness is defined as having on average relatively fewer descendants in the following generation.

The properties of underdominant polymorphisms have long been well described for a single population and are characterized by an unstable equilibrium (Fisher, 1922; Wright, 1931, 1941; Haldane, 1942; Wiener, 1942). Thus, a mutant allele that is less fit when heterozygous does not have the capacity to increase in frequency when rare and is predicted to be lost from the population. However, in an underdominant fitness configuration, an allele is predicted to proceed to fixation when starting at a frequency greater than the unstable equilibrium value, $\hat{p}$. Therefore, in a single population, underdominance sets up a type of evolutionary bi-stable switch where a population is expected to exist in one of two stable states of allele frequency, $p=0$ or $p=1$. We use + to denote a wildtype allele and $T$ to denote a genetically modified construct (or mutant allele). In a single population, the latter has frequency $p$ while the former is at frequency $1-p$. The alleles are underdominant with respect to each other if the fitness of the heterozygote, $w_{T+}$, is less than both homozygote fitnesses, $w_{T+}<w_{++}$and $w_{T+}<w_{T T}$, Fig. D.1 (a). Assuming random mating in a single population, the average fitness of the modified allele is $f_{T}(p)=w_{T T} p+w_{T+}(1-p)$ (i.e. the $T$ allele will be paired with another $T$ at frequency $p$ which will result in a fitness of $w_{T T}$; at frequency $1-p$, it will be paired with a wild type allele, leading to a fitness of $w_{T+}$ ). Equivalently, the average fitness of a wildtype allele is $f_{+}(p)=w_{++}(1-p)+w_{T+} p$. At the unstable equilibrium, with both alleles present, the two average allele fitnesses are equal to each other, $f_{T}(\hat{p})=f_{+}(\hat{p})$. At this point, $\hat{p}$, the two alleles are thus neither increasing or decreasing in frequency relative to each other. By substituting the above relationship, this predicts that the ratio of alleles at this equilibrium is equal to the inverse ratio of differences between the respective homozygotes and the heterozygote,

$$
\frac{\hat{p}}{1-\hat{p}}=\frac{w_{++}-w_{T+}}{w_{T T}-w_{T+}} .
$$

This can be rearranged into the more familiar form of

$$
\hat{p}=\frac{w_{++}-w_{T+}}{w_{++}-2 w_{T+}+w_{T T}},
$$

cf. Li (1955); Hartl and Clark (1989). Note that, without loss of generality, we set the fitness of the wildtype homozygote to one, $w_{++}=1$. All other fitnesses are positive numbers relative to this value.

Chromosomal rearrangements, such as translocations and inversions, are classic examples of potentially underdominant mutations. The offspring of heterozygous individuals are predicted to have high rates of segmental aneuploidy (disrupted number of gene copies) in their 
offspring. This can result in lethality of the offspring and thus partial sterility of the heterozygote, which reduces fitness (Snell, 1946). It has long been recognized that, with underdominance, the probability of fixation of a new, rare mutant is exceedingly small in populations of more than a few individuals (Wright, 1941; Kimura, 1962; Bengtsson and Bodmer, 1976; Lande, 1979). Yet it is clear that mutations, including translocations, with potential underdominant effects accumulate between species and are common even among some closely related species (Bush et al., 1977; White, 1978). Several models have been proposed to try to explain this discrepancy (Bengtsson and Bodmer, 1976; White, 1978; Hedrick, 1981; Walsh, 1982). This is discussed in Nachman and Searle (1995) and Rieseberg (2001).

Rather than addressing the probability of naturally establishing underdominant variants as in the "chromosomal speciation" literature cited above, we focus on artificially establishing underdominant polymorphisms and the stability of these systems once established. In contrast to the predicted loss of polymorphism in a single population, when there are migrants between multiple populations an underdominant polymorphism can be stably maintained. In the case where different neighboring populations are at high and low allele frequencies respectively, the offspring of rare migrants tend to be heterozygous. Due to their lower fitness, the rarer allele tends to be removed from the population resulting in different migration-selection equilibria in the neighboring populations. However, if migration rates between the populations are sufficiently high, the rarer allele is not removed at a sufficient rate by selection. As a result the underdominant polymorphism becomes destabilized. An analytic solution describing this critical point of destabilization, as a function of the strength of selection and the migration rate between the two demes, has been described in a fully symmetric model (Karlin and McGregor, 1972a), and for the limiting case of migration in a single direction where homozygotes are at different fitness values and the allele frequency in one of the populations is fixed at zero (Lande, 1985). Diffusion approximations describing this property have been made for the two-deme model (Barton and Rouhani, 1991). Also, a travelling wave approximation (Fisher, 1937) has been used to describe the conditions permissible for the establishment and spread, despite underdominance, of an allele in continuous habitats with a higher homozygous genotype fitness (Barton, 1979; Piálek and Barton, 1997; Soboleva et al., 2003). We do not mean to imply that underdominance is commonly responsible for the maintenance of polymorphism in natural structured populations. In addition to neutral mutations (Kimura, 1968), and mutation-selection balance (Haldane, 1924), there are many other forms of selection that can maintain polymorphism among demes, especially local adaptation, e.g., Nagylaki and Lou (2001); Lenormand (2002); Bürger (2009). Here, with underdominance, we focus on the most efficient release strategy to achieve a stable local transformation and cases with fitness and/or migration rate asymmetry.

Part of our motivation to focus on the stability of established underdominant differences is the proposal to use underdominance as a means to genetically transform wild populations with desirable alleles, e.g. to render insects resistant to diseases to prevent their transmission to humans (Curtis, 1968). In essence, releases of individuals are made that result in a population allele frequency in the wild greater than $\hat{p}$. The transformation of the population is then predicted to proceed by natural selection without additional releases or intervention. This has the desirable properties of reversibility and geographic stability. Releases of wildtype alleles resulting in a frequency lower than $\hat{p}$, Eq. (2), are predicted to ultimately remove all modified 
alleles from the wild population. Additionally, in certain situations, modified alleles are not expected to spread far beyond the initial release range nor be lost from the wild. This can be an important consideration for initial testing of refractory effector constructs (e.g., Ito et al. (2002)) in field trials and for non-native invasive disease vectors that threaten susceptible species (e.g., Warner (1968)); local populations can be stably transformed to be refractory while a wildtype state is maintained in the vectors native range. Despite intensive work to this end in the 1970-80s, using radiation induced chromosomal rearrangements, this approach ultimately failed. This is partially because the genetically modified homozygous individuals suffered from dramatically reduced fitness relative to wildtypes (e.g. Foster et al. (1972); Lorimer et al. (1972); Boussy (1988) and references therein; see also Harewood et al. (2010)). However, with new, more precise molecular genetic technologies, there is a growing interest in systems, including underdominance, that have the capacity to transform wild populations (Davis et al., 2001; Sinkins and Gould, 2006; Magori and Gould, 2006; Gould, 2008).

In the second part of the introduction we introduce a simplified symmetrical model governing the dynamics of an underdominant polymorphism in two populations of infinite size coupled by migration. We review the bifurcation pattern and the linear stability analysis that allows one to find and classify the different equilibria. Although all stable equilibria and some unstable ones can be calculated, a full phase portrait can only be obtained by supporting numerical methods. In Section 2 we give a quantitative estimate for the basin of attraction emerging in the presence of the nontrivial stable equilibria. In Section 3 we briefly discuss how the pattern in average fitness may affect the outcome of a transformation. In Section 4 we then discuss situations with broken symmetry, i.e. when there is unequal migration between the two populations and/or unequal homozygote fitnesses. Section 5 is a further discussion of our results and gives suggestions for practical applications. Finally, Section 6 serves as a summary.

\subsection{Wright-Fisher dynamics}

Here, we introduce the equations governing the dynamics of the system as it evolves from one generation to the next. This is based on the Wright-Fisher model, i.e. sampling alleles with replacement between discrete non-overlapping generations (Wright, 1931), in two coupled infinitely large populations. In the single population example, the contribution of an allele to the next generation is the allele's average fitness multiplied by its frequency in the present generation. This product is divided by the total average fitness of all alleles in the population, $\bar{w}$, to normalize it to a frequency from zero to one. Thus, the expected frequency of allele $T$ in the next generation, $p^{\prime}$, is proportional to the frequency in the present generation, $p$,

$$
p^{\prime}=\frac{f_{T}(p)}{\bar{w}} p
$$

where $\bar{w}=f_{T}(p) p+f_{+}(p)(1-p)$ is the total average fitness. Note that this average fitness is independent of the specific evolutionary model and could also be directly used in alternative systems such as the Moran model with overlapping generations.

In the two population case we have to account for migration rate, $m$, which is defined as the proportion of immigrant individuals entering a population prior to mating. This implies that 
$1-m$ is the fraction of non-migrants each generation. For symmetric interactions see Fig. D.1 (b). The genotypic fitnesses are fixed values: given the value of the reduced heterozygote fitness $w_{T+}=\omega<1$, the simplest case emerges when both homozygote fitnesses are set to one, $w_{++}=w_{T T}=1$ (Fig. D.1 (a)). The average fitness of an allele changes with its frequency: in population $i=1,2$, let $p_{i}$, and $1-p_{i}$ be the frequencies of alleles $T$ (a modified allele) and + (wildtype), respectively. The wildtype allele has average fitness $f_{+}\left(p_{i}\right)=1-$ $p_{i}+\omega p_{i}$, whereas the average modified allelic fitness amounts to $f_{T}\left(p_{i}\right)=p_{i}+\omega\left(1-p_{i}\right)$, compare to the previous subsection. Due to equal homozygote fitnesses, $w_{++}=w_{T T}=1$, we have the symmetry property $f_{T}(p)=f_{+}(1-p)$. Taking migration into account, in population $i$, the frequency of allele $T$ is $(1-m) p_{i}+m p_{j}$. As we express the average fitness of allele + in terms of the frequency of allele $T$, the total average fitness in population $i$ is

$$
\begin{aligned}
\overline{w_{i}}\left(p_{i}, p_{j}\right)= & {\left[(1-m) p_{i}+m p_{j}\right] f_{T}\left((1-m) p_{i}+m p_{j}\right) } \\
& +\left[(1-m)\left(1-p_{i}\right)+m\left(1-p_{j}\right)\right] f_{+}\left((1-m) p_{i}+m p_{j}\right),
\end{aligned}
$$

where $j \neq i$. The state of the system is characterised by the two population allele frequencies $p_{1}$ and $p_{2}\left(0 \leq p_{1}, p_{2} \leq 1\right)$, which evolve from one generation to the next as

$$
\begin{aligned}
& p_{1}^{\prime}\left(p_{1}, p_{2}\right)=\frac{\left[(1-m) p_{1}+m p_{2}\right] f_{T}\left((1-m) p_{1}+m p_{2}\right)}{\overline{w_{1}}\left(p_{1}, p_{2}\right)}, \\
& p_{2}^{\prime}\left(p_{1}, p_{2}\right)=\frac{\left[(1-m) p_{2}+m p_{1}\right] f_{T}\left((1-m) p_{2}+m p_{1}\right)}{\overline{w_{2}}\left(p_{2}, p_{1}\right)} .
\end{aligned}
$$

We now focus on the equilibrium points of the dynamics, namely we are interested in all points with $\Delta p_{i}=p_{i}^{\prime}-p_{i}=0$ for $i=1,2$, depending on $\omega$ and $m$. Some of these fixed points are independent of the parameters, others only emerge in a certain parameter range. Trivial equilibrium points are $\left(\hat{p}_{1}, \hat{p}_{2}\right)=(0,0)$ and $\left(\hat{p}_{1}, \hat{p}_{2}\right)=(1,1)$, where both populations are fixed for the wildtype or the modified allele. In the symmetric case, there is also an unstable equilibrium or a saddle point (depending on $m$ ) at $\left(\frac{1}{2}, \frac{1}{2}\right)$. These three fixed points do not change position for any pair of parameters $\omega$ and $m$. For $m=0$, the system behaves as two single populations and all nine possible combinations of fixed points exist (e.g. $\left(\frac{1}{2}, 1\right),\left(0, \frac{1}{2}\right)$, etc.). For $m>0$, we take the symmetry of the system into account. This allows for solving only one equation, for instance $\Delta p_{1}=0$. With this, we find two fixed points on the axis $\left(p_{1}, 1-p_{1}\right)$, namely $\left(\hat{p}_{1}, \hat{p}_{2}\right)=\left(x_{i}, 1-x_{i}\right), i=1,2$, where

$$
x_{1,2}=\frac{1}{2} \pm \frac{\sqrt{(1-\omega)(1-\omega-4 m)}}{2(1-\omega)(1-2 m)} .
$$

Note that with $m=0$, this reduces to the two fixed points $\left(\hat{p}_{1}, \hat{p}_{2}\right)=(1,0)$ or $(0,1)$. For $m>0$, the solution is real valued if $(1-\omega)(1-\omega-4 m) \geq 0$. The first term is always positive, but the second term becomes zero at critical value of the migration rate $m$, where a bifurcation occurs,

$$
\mu_{1}(\omega)=\frac{1-\omega}{4}
$$


The interior solutions (7) exist for $m \leq \mu_{1}(\omega)$.

A linear stability analysis of these fixed points yields a second critical point, $\mu_{2}$ (see Appendix A ), given by

$$
\mu_{2}(\omega)=\frac{1}{4}(3-\sqrt{5+4 \omega}) .
$$

Note that we have $\mu_{2}(\omega)<\mu_{1}(\omega)$ for underdominance, $\omega<1$. For $m<\mu_{1}(\omega)$, one eigenvalue of the Jacobian associated with the fixed point is negative. For $m<\mu_{2}(\omega)$, the second eigenvalue becomes negative and a new pair of stable equilibria arises. They are located on the diagonal $\left(p_{1}, 1-p_{1}\right)$ in the state space.

The bifurcation pattern is three-dimensional, given by the coordinates $\left(p_{1}, p_{2}, m\right)$ for a given heterozygote fitness $\omega$. In Fig. D.2 (a) we use a projection to the plane $(p, m)$, where $p$ simultaneously stands for $p_{1}$ and $p_{2}$. In both planes, the pattern looks the same, which is due to the symmetry of the system. Although the second bifurcation obeys this symmetry as well, we are not able to calculate its actual shape - and thus the position of the associated unstable fixed points - analytically. The set of all possible internal equilibria, from analytical predictions as well as from numerical root finding, is given in Fig. D.2 (b). Fig. D.2 (c) shows how the two bifurcation points depend on the fitness of the heterozygotes $\omega$.

In Fig. D.3 we show several slices of the $\left(p_{1}, p_{2}\right)$-plane for different pairs of parameters $(\omega, m)$; the phase portrait changes with the control parameter $m$. Note that we have $\mu_{2}(\omega)<$ $\mu_{1}(\omega)$ for $\omega<1$. For $m<\mu_{1}(\omega)$, we have $\lambda_{1}<0$. In addition for $m<\mu_{2}(\omega)$, we get $\lambda_{2}<0$. A new pair of stable internal equilibria arises. They are located on the diagonal $\left(p_{1}, 1-p_{1}\right)$ in the state space.

In Appendix B, we further discuss the system, also showing that for sufficiently low migration rates, the dynamics are well captured by a linearized system of equations. This is especially interesting for further analytical examinations that go beyond the scope of this manuscript, e.g. when a larger system of interacting populations is considered.

Taking advantage of the symmetry in the system of two populations coupled by migration allows one to analytically calculate the emergence of stable internal equilibria depending on the heterozygote's fitness $\omega$. With the additional aid of numerical methods we can also find the saddle points. In the next section we discuss the pattern in more detail, addressing the problem of finding the basins of attraction that belong to the internal stable equilibria.

In Appendix C, we present results when considering three alleles with underdominance, which necessarily becomes more complicated and has implications for transformation strategies. However, in certain situations bi-stable population transformations are still possible, even if underdominance is not present between all pairs of alleles. Also, in the two population case, even at the non-trivial stable equilibria, if only underdominance is present, alleles are expected to be lost and the system returns to the two-allele case. This suggests that in certain contexts the results for a two-allele system are applicable to systems with initially more than two alleles. 


\section{The basin of attraction}

What is the region in the state space where the system is attracted to a non-trivial stable equilibrium? Once the two non-trivial stable equilibria are present, i.e. for $0<m<\mu_{2}(\omega)$ there is a region where the system, once initiated there, will ultimately reach one of the stable equilibria along $\left(x_{i}, 1-x_{i}\right)$, Eq. (7). This region is the basin of attraction. Here, we give two estimates of this subset of the state space and compare it to simulations.

Consider the boundaries of the system. In one population the frequency of the transgenic type is fixed to zero or one, and in the other it can have any value between zero and one. Due to the symmetry in homozygote fitnesses and migration rate, we only have to consider those parts of the boundaries where for instance $p_{2}=0$, as in this case $p_{2}=1$ follows directly from that.

In the neighborhood of the boundaries, we expect the components of the (two-dimensional) flow parallel to the boundaries to be similar to the (one-dimensional) flow along the boundaries. Wherever the flow along the boundaries vanishes there are points of vanishing flow of the quasi-one-dimensional system. They can be stable or unstable, depending on the slope of the one-dimensional flow at these points, $\partial_{p} \Delta p_{1}(p, 0)$, or $\partial_{p} \Delta p_{2}(0, p)$. Their position will give an estimate of where the basin of attraction meets the boundaries, assuming low migration. Consequently, we need the solutions of the one-dimensional system, $\Delta p_{1}(\rho, 0)=\Delta p_{2}(0, \rho)=0$, which are given by

$$
\rho_{ \pm}=\frac{3-m(1-\omega)-3 w \pm \sqrt{(1-\omega)\left(1+m^{2}(1-\omega)-2 m(3+\omega)-\omega\right)}}{4(1-m)(1-\omega)} .
$$

If both equilibria exist $\rho_{-} \leq \rho_{+}$. Additionally, $\left(\rho_{-}, 0\right)$ is unstable, since $\left.\partial_{p} \Delta p_{1}(p, 0)\right|_{p_{=} \rho_{-}}>0$ and hence $\left(\rho_{-}, 0\right)$ is a naïve minimum of a possible initial condition at the boundary that leads to the stable equilibrium $\left(x_{2}, 1-x_{2}\right)$, compare Eq. (7) and Fig. D.4. Thus, possible estimates for the regions where the dynamics leads to one of the stable equilibria given by the coordinates $\left(x_{i}, 1-x_{i}\right)$, Eq. (7), for $0 \leq m<\mu_{2}(\omega)$, are rectangles, for example the one limited by the four points $\left(\rho_{-}, 0\right),(1,0),\left(1,1-\rho_{-}\right)$and $\left(\rho_{-}, 1-\rho_{-}\right)$, cf. Fig. D.4. At high migration rates this increasingly underestimates the basin. In addition, we can give another estimate, which is the deltoid (kite) limited by the four points $\left(\frac{1}{2}, \frac{1}{2}\right),\left(1,1-\rho_{-}\right),(1,0)$, and $\left(\rho_{-}, 0\right)$. For low migration this in an overestimate of the basin of attraction, which itself is obtained by numerical simulations, see Fig. D.4.

\section{Average fitness}

Do the patterns of reduced average fitness affect the strategy for achieving transformation? For the symmetric model, the mean population fitness at the central unstable equilibrium can be found by substituting $\hat{p}_{i}=\frac{1}{2}$ into Eq. (4). This results in $\overline{w_{i}}=(1+\omega) / 2$. Near this point is the optimal strategy for transforming a target population with a minimum release of individuals (see Section 5). However, this is also the point where mean population fitness is at its lowest. At the approximate threshold for transformation with a single population release,

$$
\left(p_{1}, p_{2}\right)=\left(\rho_{-}, 0\right)
$$


the average fitness in each population is

$\overline{w_{i}}\left(\rho_{-}, 0\right)=\frac{1}{4}(1-m)\left(3-m+\omega(1+m)-\sqrt{(1-\omega)\left((1-\omega)\left(1+m^{2}\right)-2 m(3+\omega)\right)}\right)$,

which only results in a small increase in mean fitness except at very high migration rates, see Fig. D.5, where the approximation is less accurate and stability of the system is almost lost. Thus, the strategy to minimize release numbers probably has more advantages than maximizing average population fitness during the transformation.

Interestingly, the mean population fitness at the stable non-trivial fixed point, where the system is in migration-selection equilibrium, appears to be independent of the heterozygote fitness, $\omega$. This point is given by $\hat{p}_{1,2}=x_{1,2}$, Eq. (7). The point is stable for $0<m<\mu_{2}$, Eq. (9). Substituting this into Eq. (4) yields $\overline{w_{i}}=1-2 m$, which is only a function of the migration rate. Intuitively, if heterozygotes are less fit then the rarer allele will have a lower frequency, at equilibrium, in the population, producing fewer heterozygotes each generation. Conversely, if heterozygotes are more fit the rarer allele will attain a higher frequency producing more heterozygotes each generation. The number of heterozygotes produced and their fitness cancel out in terms of the average effect in the population. This is similar to the effects of deleterious mutations at mutation-selection equilibrium, in which case the average fitness of a population is only a function of the mutation rate (Haldane, 1937).

\section{Unequal homozygote fitnesses and non-symmetric migration}

So far all results are based on the symmetry of the system of two coupled populations with an underdominant locus. In this section, we show how the phase portrait changes for non-symmetric migration between the two populations and if restriction of the model to equal homozygote fitnesses is relaxed, see Fig. D.1 (c). We allow migrants in one population, coming from the other, to have an abundance $m_{i}$, with $m_{1} \neq m_{2}$ in general, see Fig. D.1 (d). For example, migration bias can arise between upstream (or upwind) and downstream (downwind) populations, in the direction of an invasive front with a demographic expansion, or when migration occurs between populations of unequal size. Typically, the genetically modified type suffers from a fitness reduction compared to the wildtype with a fitness of one (Boussy, 1988). This reduction results in a lower fitness $w_{T T}=\nu$, with $0<\omega<\nu \leq 1$. Discarding the simplifying assumptions that lead to a high degree of symmetry disrupts the symmetrical arrangement of the phase portrait. As a result, the critical values in parameter space and thus the stable equilibria can no longer be calculated analytically.

With $w_{+T}=\omega, w_{T T}=\nu$, and $w_{++}=1$, the respective average allelic fitnesses in population $i$ are

$$
\begin{aligned}
& f_{+}\left(p_{i}\right)=1-p_{i}+\omega p_{i}, \\
& f_{T}\left(p_{i}\right)=\nu p_{i}+\omega\left(1-p_{i}\right),
\end{aligned}
$$

for wildtype + and modified type $T$. Note that both fitness functions are linear in $p$, but in general $f(m p) \neq m f(p)$. Nevertheless, observe that $f\left((1-m) p_{i}+m p_{j}\right)=(1-m) f\left(p_{i}\right)+$ 
$m f\left(p_{j}\right)$ holds for all values of $\omega$ and $\nu$. More importantly, $f_{T}(p)$ equal to $f_{+}(1-p)$ no longer holds either, which is due to loss of symmetry in homozygote fitness. Similar to Eq. (4) the average fitness in population $i$ thus reads

$$
\begin{aligned}
\overline{w_{i}}\left(p_{i}, p_{j}\right)= & \left(1-m_{i}\right)^{2}\left[p_{i} f_{T}\left(p_{i}\right)+\left(1-p_{i}\right) f_{+}\left(p_{i}\right)\right] \\
& +m_{i}\left(1-m_{i}\right)\left[p_{i} f_{T}\left(p_{j}\right)+\left(1-p_{i}\right) f_{+}\left(p_{j}\right)\right] \\
& +\left(1-m_{i}\right) m_{i}\left[p_{j} f_{T}\left(p_{i}\right)+\left(1-p_{j}\right) f_{+}\left(p_{i}\right)\right] \\
& +m_{i}^{2}\left[p_{j} f_{T}\left(p_{j}\right)+\left(1-p_{j}\right) f_{+}\left(p_{j}\right)\right]
\end{aligned}
$$

where $j \neq i$. As we consider infinitely large populations with random mating taking place after migrants are exchanged, $m_{j}$ neither gives a contribution to $\overline{w_{i}}$, nor does it directly influence the frequency of allele $T$ in the next generation, $p_{i}^{\prime}$ :

$$
\begin{aligned}
& p_{1}^{\prime}\left(p_{1}, p_{2}\right)=\frac{\left(1-m_{1}\right)^{2} p_{1} f_{T}\left(p_{1}\right)+\left(1-m_{1}\right) m_{1}\left(p_{1} f_{T}\left(p_{2}\right)+p_{2} f_{T}\left(p_{1}\right)\right)+m_{1}^{2} p_{2} f_{T}\left(p_{2}\right)}{\overline{w_{1}}\left(p_{1}, p_{2}\right)} \\
& p_{2}^{\prime}\left(p_{1}, p_{2}\right)=\frac{\left(1-m_{2}\right)^{2} p_{2} f_{T}\left(p_{2}\right)+\left(1-m_{2}\right) m_{2}\left(p_{1} f_{T}\left(p_{2}\right)+p_{2} f_{T}\left(p_{1}\right)\right)+m_{2}^{2} p_{1} f_{T}\left(p_{1}\right)}{\overline{w_{2}}\left(p_{2}, p_{1}\right)} .
\end{aligned}
$$

From the shift in fixed points and rate of allele frequency change, when considering a phase portrait (Fig. D.6) we can see that unequal migration rates result in a loss of bilateral symmetry. Unequal homozygote fitnesses result in a loss of rotational symmetry. With both of these effects, all forms of symmetry are lost as illustrated in Fig. D.6, (IV). Furthermore, from this asymmetry it becomes apparent that situations exist where a stable, local transformation of a less fit allele is possible in only one of the two coupled populations. The stability properties under symmetry distortion can be represented in a "phase diagram" Fig. D.7. From this example it can also be seen that both unequal migration rates and unequal homozygote fitnesses are required to result in non-trivial stability in only one of the two populations. In this doubly asymmetric case, higher migration rates and lower homozygote fitnesses can result in single population stability outside the range of parameter values necessary for symmetric stability in two populations: e.g. the lower right tip of zone B in Fig. D.7 has a lower homozygote fitness and a higher migration rate than is found for any area of zone A. In this regime, lower homozygote fitnesses are counterbalanced to some degree by higher emmigration and lower immigration rates.

As homozygote fitnesses become more asymmetrical, the non-trivial stable fixed points move closer to the boundaries of the system. Solving (16) for the edge of the system, $p_{2}=0$, as done in Section 2 for the fully symmetrical model, and assuming stationarity, $\Delta p_{1}=0$, gives the non-trivial solutions

$$
\rho_{ \pm}=\frac{2+\nu(1-m)-\omega(3-m) \pm \sqrt{(\nu-\omega)^{2}(1+m)-2 m\left(2 \nu(1-\omega)+\nu^{2}-\omega^{2}\right)}}{2(1-m)(2 \omega-\nu-1)}
$$


where $\rho_{+}$is stable and $\rho_{-}$is the unstable internal fixed point in the one-dimensional system. Setting $\rho_{-}=\rho_{+}$, the point where stability is lost according to the flow along the edge (when the argument in the square root is zero), gives

$$
\mu_{3}(\omega, \nu)=\frac{\nu(2-2 \omega+\nu)-\omega^{2}-2 \sqrt{\nu(1+\nu-2 \omega)\left(\nu-\omega^{2}\right)}}{(\nu-\omega)^{2}} .
$$

This is an approximation of the critical migration rate allowing stability in the case of unequal homozygote fitnesses. As can be seen in Fig. D.8 this edge-approximation works very well when $\nu<(\omega+1) / 2$ or when the transgenic homozygote fitness is less than the average of the heterozygote and wildtype fitnesses. Above this area there is an increasing deviation as the symmetric critical migration rate $\mu_{2}$ is approached. An ad hoc non-linear weighted average between $\mu_{2}$ and $\mu_{3}$ of

$$
\mu_{\mathrm{w}}(\omega, \nu)=\mu_{3}(\omega, \nu)(1-\nu)+\frac{\mu_{2}(\omega)(\nu-\omega)^{2}}{(1-\omega)^{2}} .
$$

gives a good fit across the entire range.

It can also be casually observed in Fig. D.6 that the central unstable equilibrium always falls along the $p_{1}=p_{2}$ axis despite various forms of parameter asymmetry. Solving Eqs. (16) and (17) for an unstable fixed point along this axis (similar to Appendix A) yields

$$
\hat{p}_{1}=\hat{p}_{2}=\frac{1-\omega}{1-2 \omega+\nu} .
$$

Thus, the central unstable equilibrium is independent of migration and identical to the unstable equilibrium in the single population case, Eq. (2). This makes intuitive sense if one considers that this is also the stable equilibrium point in the case of overdominance (i.e. heterozygote advantage) and that at this equilibrium all populations should arrive at the same allele frequency. At this point the migrants between populations have no effect on changing allele frequencies.

\section{Discussion}

At high and very low migration rates the dynamics of the two population system approaches that of a single population system, either as two independent populations or as a single combined population. Here we have explored the interesting cases between these two extremes where the dynamics are more complex. A two-population single-locus system can have up to nine equilibrium points, two of which can be non-trivially stable. We have focused on the conditions of this non-trivial stability and how the system can arrive at these points. First however, it is important to be clear about the assumptions and limitation of the necessarily simplified model.

The system described here assumes selection acting on a single locus, which is appropriate for certain kinds of chromosomal rearrangements, such as paracentric inversions and fusions, discussed by Lande (1979), and single gene effects, similar to Rh factor, e.g. (Wiener, 1942). Yet, in general the biological examples provided for underdominance are reciprocal 
chromosomal translocations, which of course involves two (or more) loci. In plants, fungi and protists that undergo alternation of generations where diploid stages are separated by multicellular haploid gametophytes, unbalanced translocations can be lethal at the gametophyte stage (Ray et al., 1997). In this simple case of complete lethality, the system behaves as a single locus with $\omega=\frac{1}{2}$ (Wright, 1941). In typical animals, gametes that have an unbalanced set of chromosomes can function normally and produce a zygote (Snell, 1946). Thus, there is a small chance that two unbalanced gametes could complement each other upon fertilization, so unbalanced zygotic lethality in animals can behave as a single locus with $\omega$ slightly greater than $\frac{1}{2}$ (Wright, 1941). Of course, even with full unbalanced lethality, other factors in various species such as competition among offspring, parental care, some fraction of vegetative reproduction, and/or alternate chromosome segregation patterns can result in an effective $\omega$ substantially greater than one half (Lande, 1979). However, if an organism with an unbalanced translocation can survive to reproduce, as is perhaps the case with some human diseases and translocations that involve smaller chromosomal regions e.g. (Koochek et al., 2006), then the system can no longer be expected to behave according to the single locus model presented here.

Another important limitation is the simplifying assumption of an infinitely large population size, where only migration and selection are the sole determinants of allele frequency change. A finite population will ultimately reach the absorbing states at $p_{1}=p_{2}=0$ or $p_{1}=p_{2}=1$. However, the time until these points are reached may be very large, in particular when the corresponding deterministic system has stable interior fixed points and selection is relatively strong. An interesting question in this case is how long until a local transformation is lost and, in the case of asymmetry, what are the relative likelihoods of ultimate fixation for the wildtype versus transgenic alleles. This is briefly presented in Appendix D.

If a stable transformation of a population is possible, from an applied point of view, $\rho_{-}$ from Eq. (10) gives the approximate minimum frequency that must be surpassed to result in a stable transformation, by releasing the transgenic construct into a single target population. In order to attain a target frequency $p^{*}$, releases must be made of size $P=\left(p^{*} /\left(1-p^{*}\right)\right)$ relative to the wild population (i.e. after the release of $P$ individuals homozygous for a modified allele into a wildtype population of relative size 1 , the allele frequency becomes $p^{*}=P /(1+P)$ ). This function increases steeply towards positive infinity as $p^{*} \rightarrow 1$, thus small differences in $p^{*}$ can have large effects and, for practical reasons, larger $p^{*}$ s should be avoided. Because of this, stable transformations might be more efficiently achieved with smaller, asymmetrical releases into both populations (i.e. closer to the central unstable equilibrium point, which is at the tip of all basins of attraction, Fig. D.4). For example, starting at $p_{1}=p_{2}=0$, a total of a two fold equivalent of a single wildtype population is required for equal releases into two populations to result in the central unstable point, $p_{1}^{*}=p_{2}^{*}=\frac{1}{2}$ (with equal homozygote fitness). While a larger minimum of a four fold equivalent is required to enter the basin of attraction by a single population release, if for example the threshold, Eq. (10), is at $p^{*}=0.8$. Specifically, the proposed minimal-number strategy is a $p^{*}$ close to, but less than, $\hat{p}$, Eq. (2), in non-target, neighbouring populations, and a $p^{*}$ close to, but greater than, $\hat{p}$ in the target population. Estimating the central equilibrium point has the advantage of less uncertainty due to its independence from rates of migration, see Section 4. However, the basin near the central equilibrium is a smaller target area and entering near there raises the risk of accidentally 
transforming both populations or only the non-target population. If this is an undesirable outcome, it must also be considered. These calculations naïvely assume released individuals are equivalent to wildtype in terms of mating success and that both sexes will be released. In reality it is likely that released individuals are less fit and may even be discriminated against by wild female mate choice (Lance et al., 1998; McInnis et al., 1996), and that (in many disease vectoring insect species) releases of only males would be made necessitating more than one generation of release to achieve $p^{*}>\frac{1}{2}$. However, the basic strategy of more efficient transformations closer to the central equilibrium remains unchanged.

Sterile insect technique (SIT) is a widely used and in some cases very successful genetic pest management (GPM) approach where the goal is suppression and elimination of the wild population, rather than transforming the population, and incidentally often requires larger release sizes than those predicted here for underdominant transformation (Asman et al., 1981; Krafsur, 1998). However, SIT can be less effective in species that have high density-dependant population size regulation such as mosquitoes, i.e. a high reproductive potential allowing a rapid rebound from a small number of individuals (Prout, 1978; Dye, 1984). In contrast, underdominant mediated population transformation may have advantages in species with high density-dependant regulation. Part of the original interest in underdominance was due to its potential population suppression effects, similar to SIT, rather than population replacement, e.g. Vanderplank (1944); Laven (1969). This population suppression is greatest near unstable equilibriums where the mean population fitness is minimized (Serebrovskii, 1940). One potential problem in a species with low density dependence is that during an underdominant transformation the wild population size may start to decline as the populations transits through low average fitnesses near unstable equilibria (see Section 3). This population size effect is dependent on additional fecundity parameters not modelled here, but it is easy to imagine that the target population may become more susceptible to immigration from other wild populations during this phase (similar to a "migrational meltdown," Ronce and Kirkpatrick (2001); Tufto (2001)). Hence, it may be difficult to locally transform certain species in such a case. However, in species with high reproductive potential, where only a few individuals can quickly produce enough descendants to return to carrying capacity, this should not be as large of an issue because the population is continuously maintained near carrying capacity. This is precisely the situation where SIT can be very ineffective. Thus, underdominant mediated population transformation is an excellent alternative to SIT in GPM strategies because the two approaches are likely most effective at opposite ends of the density-dependent spectrum. Note also that at mid-spectrum the two methods might be usefully combined to first reduce the wild population by SIT, followed by population replacement by underdominance.

\section{Summary}

In summary, Section 1 introduces the problem and gives the criteria permissible to a stable transformation in a single target population, which is a function of the migration rate, Eq. (9). This is based on a stability analysis (Appendix A). Appendix B, extends this approach to a simplified linear treatment of the dynamics, which can be useful in more complicated and realistic population models beyond the simple two-deme system analysed here. In Appendix C 
we illustrate underdominant dynamics for more than two alleles, which in some cases is similar to the expectations of a two-allele system.

In Section 2 we identify the full basin of attraction numerically and approximate it analytically. For a stable local transformation, this is important when considering release strategies.

In Section 3 we determine the effect of a local population transformation on the average fitness of the population. Counter-intuitively we find that average fitness is independent of the genotypic fitness parameters and only a function of the migration rate.

In Section 4 we analyse cases of asymmetric fitness and/or asymmetric migration rates, which generally act to destabilize the system. Initially surprising results are that there are cases where stable-local transformations are only possible in one of the populations and the central unstable equilibrium is independent of migration. We also derive an approximation for critical migration rates permissive to stability with asymmetric fitness, Eq. (19).

The expected sensitivity to fecundity of underdominance complements alternative population management techniques. This, and other consequences of the model for practical use are discussed in Section 5: In general there is very little difference in fitness for alternative strategies to enter the basin of attraction. In future studies of finite populations the fecundity of the organism can be included in the model to directly quantify this effect.

Finally, in Appendix D we give numerical results from simulations of stable transformations in finite populations. We find that if effective population sizes are large $(\approx 100$ diploid individuals) and the system is not near critical boundaries (migration rates and heterozygote fitnesses are relatively low) a local transformation can remain stable for a very long time (for more than $10^{4}$ generations). If homozygotes for the modified allele are only slightly less fit than the wildtype allele, the finite system is likely to ultimately result in loss of the modified allele from both populations, rather than fixation in both.

\section{Acknowledgement}

We thank C. Bank, N. Barton, P. Pfaffelhuber, L. M. Turner and three anonymous reviewers for suggestions and discussion. P. M. A. and A. T. acknowledge financial support from the DFG by the Emmy-Noether program. R. G. R. and F. A. R. are supported by funds from the Max-Planck Society.

\section{Appendix A. Linear stability anaysis}

A system similar to Eqs. (4)-(6) can be found in Karlin and McGregor (1972a) (see also Karlin and McGregor (1972b)), where a linear stability analysis has also been performed. Here we briefly repeat this procedure. Additionally, this formalism can also be applied to the linearized system, (Appendix B), and used to solve for the central unstable equilibrium along the $\left(x_{i}, x_{j}\right)$ axis in the asymmetric model (Section 4$)$.

Linear stability analysis reveals whether a fixed point of a dynamical system is attracting or repelling (Hofbauer and Sigmund, 1998; Strogatz, 2000; Murray, 2007). The idea is the following: given an equilibrium point, if we perturb the system, will it move away from that point or return to it? This property is expressed by the eigenvalues of the Jacobian matrix at the 
equilibrium point $\left(\hat{p}_{1}, \hat{p}_{2}\right),\left.\mathbb{J}\right|_{\left(\hat{p}_{1}, \hat{p}_{2}\right)}$. The Jacobian matrix is the matrix of all partial derivatives,

$$
\mathbb{J}(x, y)=\left(\begin{array}{ll}
\left.\frac{\partial \Delta p_{1}}{\partial p_{1}}\right|_{(x, y)} & \left.\frac{\partial \Delta p_{1}}{\partial p_{2}}\right|_{(x, y)} \\
\left.\frac{\partial \Delta p_{2}}{\partial p_{1}}\right|_{(x, y)} & \left.\frac{\partial \Delta p_{2}}{\partial p_{2}}\right|_{(x, y)}
\end{array}\right) .
$$

where $\Delta p_{i}=p_{i}^{\prime}-p_{i}$, see Eqs. (5), (6). Assuming that the perturbation is small and that the Jacobian does not vanish, we obtain a linear equation that governs the temporal evolution of that perturbation. Its solutions can be written as a superposition of eigenmodes, $\propto e^{\lambda_{k} t}$, where $\lambda_{k}$ is the $k$ th eigenvalue of the Jacobian. Thus, a perturbation will become smaller over time if all eigenvalues are negative. As soon as a single eigenvalue is positive, the perturbation increases and the corresponding equilibrium point is not stable. The eigenvalues govern the behavior of the system in a vicinity of an equilibrium point (Strogatz, 2000). The explicit form of our Jacobian at $\left(x_{i}, 1-x_{i}\right)$, Eq. (7), is

$$
\mathbb{J}\left(x_{1}, 1-x_{1}\right)=\left(\begin{array}{cc}
\frac{\omega-1-m(w-6)-6 m^{2}}{(1-2 m)^{2}} & \frac{m(2 m+\omega)}{(1-2 m)^{2}} \\
\frac{m(2 m+\omega)}{(1-2 m)^{2}} & \frac{\omega-1-m(w-6)-6 m^{2}}{(1-2 m)^{2}}
\end{array}\right),
$$

which is the same for $i=1$ or 2 , due to the symmetry of the system. The eigenvalues are the solutions of the characteristic polynomial $\operatorname{det}(\mathbb{I}-\lambda \mathbb{I})=0$. They are given by

$$
\begin{aligned}
& \lambda_{1}=-\frac{1-\omega-4 m}{1-2 m} \\
& \lambda_{2}=-\frac{1-\omega+2 m(2 m-3)}{(1-2 m)^{2}}
\end{aligned}
$$

Both roots are always real valued. A local bifurcation occurs when a parameter change causes the stability of an equilibrium to change, such that new equilibria can arise. Stability changes when the $\lambda_{k}$ change signs. Thus, we can compute the critical points from $\lambda_{1}=0$, and from $\lambda_{2}=0$. In the former case, we obtain $\mu_{1}(\omega)=(1-\omega) / 4$. The latter case yields the critical point of the second bifurcation,

$$
\mu_{2}(\omega)=\frac{1}{4}(3-\sqrt{5+4 \omega})
$$

where we can neglect the second branch of the root due to consistency.

\section{Appendix B. Linearized dynamics}

The original symmetric system, Eqs. (5) and (6), is of the shorthand form $p_{i}^{\prime}\left(p_{1}, p_{2}\right)=$ $\varphi_{i}\left(p_{1}, p_{2}\right) / \overline{w_{i}}\left(p_{1}, p_{2}\right)$. A fixed point of the dynamics occurs if $\Delta p_{i}\left(p_{1}, p_{2}\right)=p_{i}^{\prime}\left(p_{1}, p_{2}\right)-$ $p_{i}=0$. The position of fixed points and the stability properties do not change if we examine $\delta p_{i}\left(p_{1}, p_{2}\right)=\Delta p_{i}\left(p_{1}, p_{2}\right) \overline{w_{i}}\left(p_{1}, p_{2}\right)=\varphi_{i}\left(p_{1}, p_{2}\right)-\overline{w_{i}}\left(p_{1}, p_{2}\right) p_{i}\left(p_{1}, p_{2}\right)$. This condition is 
quadratic in the control parameter $m$ and can be rearranged as $(i \neq j)$

$$
\begin{aligned}
\delta p_{i}\left(p_{1}, p_{2}\right)= & (1-\omega) p_{i}\left(3 p_{i}-2 p_{i}^{2}-1\right) \\
& -\left(4\left(1-p_{i}\right) p_{i}-\omega\left(1-2 p_{i}\right)^{2}\right)\left(p_{i}-p_{j}\right) m \\
& +(1-\omega)\left(1-2 p_{i}\right)\left(p_{i}-p_{j}\right)^{2} m^{2}
\end{aligned}
$$

Note that only the relative coordinate, $\left(p_{i}-p_{j}\right)$, scales with $m$. The above set of equations has exactly the same fixed points as the system (5), (6). However, the Jacobian matrix and its Eigenvalues are of different form. Namely, the latter now read

$$
\begin{aligned}
& \hat{\lambda}_{1}=-1+\omega+4 m, \\
& \hat{\lambda}_{2}=-\frac{1-\omega+2 m(2 m-3)}{1-2 m},
\end{aligned}
$$

i.e. $\hat{\lambda}_{i}=(1-2 m) \lambda_{i}$, compare Eqs. (A.3) and (A.4). With $\hat{\lambda}_{1,2}=0$ solved for $m$, this leads again to the critical points from Appendix A, see also Eqs. (8) and (9).

Up to linear order in $m$ we have approximately $\delta p_{i} \approx \delta^{L} p_{i}\left(p_{1}, p_{2}\right)=(1-\omega) p_{i}\left(3 p_{i}-\right.$ $\left.2 p_{i}^{2}-1\right)-\left(4\left(1-p_{i}\right) p_{i}-\omega\left(1-2 p_{i}\right)^{2}\right)\left(p_{i}-p_{j}\right) m$, such that $\delta^{L} p_{i}\left(p_{1}, p_{2}\right)=0$, solved along the diagonal $\left(p_{1}, 1-p_{1}\right)$ leads to the pair of internal equilibria $\left(\hat{p}_{1}, \hat{p}_{2}\right)=\left(y_{i}, 1-y_{i}\right)$ given by

$$
y_{1,2}=\frac{1}{2} \pm \sqrt{(1-4 m)} \frac{\sqrt{(1-\omega)(1-\omega-4 m)}}{2(1-\omega)(1-2 m)},
$$

which only differ from Eq. (7) by the factor of $\sqrt{1-4 m}$ in the second term. The Eigenvalues of the Jacobian $\mathbb{J}\left(y_{i}, 1-y_{i}\right)$, computed similar to Appendix A, read

$$
\begin{aligned}
& \tilde{\lambda}_{1}=\hat{\lambda}_{1}=-1+\omega+4 m, \\
& \tilde{\lambda}_{1}=\frac{1+16 m^{2}-2 m(4-\omega)-\omega}{1-4 m} .
\end{aligned}
$$

The condition $\tilde{\lambda}_{1}=0$ yields (once more) $\tilde{\mu}_{1}(\omega)=\mu_{1}(\omega)=(1-\omega) / 4$ for the first critical point. Moreover, $\tilde{\lambda}_{2}=0$ gives

$$
\tilde{\mu}_{2}(\omega)=\frac{1}{16}(4-\omega-\sqrt{\omega(8+\omega)})
$$

such that if migration rate is lower than $\tilde{\mu}_{2}(\omega)$, we observe stable internal fixed points of the dynamics described by Eq. (B.1) up to linear order. In Fig. D.2 (c) we compare the second critical point of the original system with it's equivalent from the approximation linear in $m$. Note that for $\omega<1$, we have $\mu_{2}(\omega)<\tilde{\mu}_{2}(\omega)$, whereas $\tilde{\mu}_{2}(\omega)-\mu_{2}(\omega) \rightarrow 0$ for $\omega \rightarrow 1$. If $\omega$ is above 0.012 , the relative error $\left|\tilde{\mu}_{2}-\mu_{2}\right| /\left(\tilde{\mu}_{2}+\mu_{2}\right)$ is smaller than ten percent, i.e. the prediction of stable internal equilibria is relatively robust to cutting away information on higher order migration effects. 


\section{Appendix C. Three alleles}

Here we introduce a third allele, $R$, to the system at frequency $q$. Thus, the frequency of the original wildtype allele now becomes $1-p-q$. In the most general case the $R R$ homozygote has a unique homozygote fitness of $\nu_{R R}$ and we now write the TT homozygote fitness as $\nu_{T T}$ relative to a wildtype homozygote fitness of one. The corresponding heterozygote fitnesses are $\omega_{+T}, \omega_{+R}$, and $\omega_{R T}$.

The average fitnesses of the three alleles can be written as

$$
\begin{aligned}
& f_{T}=\nu_{T T} p+\omega_{R T} q+\omega_{+T}(1-p-q) \\
& f_{+}=(1-p-q)+\omega_{+T} p+\omega_{+R} q \\
& f_{R}=\nu_{R R} r+\omega_{R T} p+\omega_{R+}(1-p-q)
\end{aligned}
$$

In general, the expected frequency of the $i^{\text {th }}$ allele in the next generation, $p_{i}^{\prime}$, is the product of the alleles frequency $p_{i}$ and fitness $f_{i}$ normalized by the sum of this product over all alleles,

$$
p_{i}^{\prime}=\frac{f_{i}}{\sum_{j} p_{j} f_{j}} p_{i} .
$$

With three alleles, the fixed points along the edges of the simplex, where the frequency of one allele is zero, is equivalent to the corresponding two allele case presented above. The remaining internal fixed point can be found by setting the three averages fitnesses equal to each other and solving the set of simultaneous linear equations. This gives the coordinates of the central unstable (assuming underdominance) equilibrium as

$$
\hat{p}=\frac{1}{\alpha} \nu_{R}\left(\omega_{T+}-1\right)+\omega_{R+}\left(\omega_{R+}-\omega_{T+}-\omega_{R T}\right)+\omega_{R T}
$$

and

$$
\hat{q}=\frac{1}{\alpha} \omega_{T+}^{2}+\nu_{T}\left(\omega_{R+}-1\right)+\omega_{R T}-\omega_{T+}\left(\omega_{R+}+\omega_{R T}\right)
$$

where

$$
\begin{aligned}
\alpha= & \omega_{T+}^{2}-\nu_{R}-\nu_{T}\left(1+\nu_{R}-2 \omega_{R+}\right) \\
& +2 \omega_{T+}\left(\nu_{R}-\omega_{R+}-\omega_{R T}\right) \\
& +\left(\omega_{R+}-\omega_{R T}\right)^{2}+2 \omega_{R T}
\end{aligned}
$$

which exists if both $\hat{p}>0, \hat{q}>0$ and $\hat{p}+\hat{q}<1$.

If there is three-way underdominance between all alleles, the minimum transformation threshold for the modified allele can be lower than if a pair of alleles are considered individually, Figure D.9A. In terms of transformation strategies, if a linked effector gene results in a substantial fitness cost, a population transformation might be more achievable by a two step process, first transforming the population with a sightly less fit modified allele; then transforming the modified population with a combined underdominant-effector construct. Similarly, if 
there are multiple wildtype alleles that together have a heterozygous fitness advantage, the transformation threshold is higher than if it is inferred with respect to a single wildtype allele, Figure D.9B. Regardless of how many alleles are in underdominance with respect to each other, in this system, fixation of the modified allele $p=1$ is stable if its homozygous fitness is greater than both of its heterozygote fitnesses, $\nu_{T}>\omega_{T+}$ and $\nu_{T}>\omega_{R T}$, and bistability with wildtype can be maintained even if there is no underdominance with respect to the third allele. This is illustrated in Figure D.9C and D.9D.

Three alleles predict a maximum of seven fixed points in a single population. This predicts a maximum of $7^{2}$ fixed points, when migration rates are low, in a coupled two-deme system. Nine of these points can be stable equilibria (three are trivial fixations), one central point is unstable and all others are saddle points, if they exist for a given migration rate. Since a two-dimensional simplex exists for each population $\left(\mathcal{S}^{2}\right)$ the joint state space for the coupled populations is four-dimensional $\left(\mathcal{S}^{2} \times \mathcal{S}^{2}\right)$; however the state space is not simply a pentachoron simplex $\left(\mathcal{S}^{4}\right.$, a four-dimensional triangle) but exists over a broader area. This is because the allele frequencies, when considered jointly for both populations, can be at any position within their respective $\mathcal{S}^{2}$ s (also, allele frequencies do not sum to one when considered jointly for both populations). This is analogous to the simpler case in Fig. D. 3 where an $\mathcal{S}^{1} \times \mathcal{S}^{1}$, for two alleles in two populations, exists on a two-dimensional square rather than an $\mathcal{S}^{2}$ triangle. If a dimension is assigned to each allele frequency in each population, we can say the $\mathcal{S}^{1} \times \mathcal{S}^{1}$ coupled system is in a plane across $\mathbb{R}^{4}$ rather than the $\mathcal{S}^{2}$ plane in $\mathbb{R}^{3}$, but both are twodimensional. Similarly $\mathcal{S}^{2} \times \mathcal{S}^{2}$ is flat across $\mathbb{R}^{6}$ rather than $\mathcal{S}^{4}$ in $\mathbb{R}^{5}$, but both $\mathcal{S}^{2} \times \mathcal{S}^{2}$ and $\mathcal{S}^{4}$ are four dimensional.

Even with three-way pairwise underdominance, at a stable migration-selection equilibrium there can not be more alleles present than the number of populations (a detailed argument is given in Karlin and McGregor (1972a)), so even if migration rates in the two-deme model are permissive to stable maintenance of polymorphism, one of the three alleles is expected to be lost. Thus, starting from the point of view of a fully underdominant system in stable equilibrium, the critical migration rates derived for the two-allele case are applicable even if additional underdominant alleles are possible. To illustrate three alleles in two populations we modify $p$ and $q$ in Eqs. (C.1), (C.2), and (C.3) to read, after migration, as $p_{1}=(1-m) p_{1}+m p_{2}$ and $q_{1}=(1-m) q_{1}+m q_{2}$ for the first population and the corresponding terms are also written for allele frequencies in second population, where, for example, $p_{1}$ is the frequency of $p$ in population 1 , and $m$ is the fraction of migrants each generation as given previously for the two-allele case. The stable maintenance of two alleles (in underdominance) in two coupled populations for this three allele system is illustrated in Fig. D.10.

\section{Appendix D. Fluctuations}

Ultimately, in populations of finite size, the concept of stability is not applicable any more. The only two absorbing states are complete fixation or complete loss of one of the alleles. However, with underdominance, the loss of polymorphism in coupled populations may take a very long time if population sizes are large and selection is very strong relative to migration. For a given set of parameter values, if a polymorphism is stable in infinite populations, we can ask the question of of how long until polymorphism is lost in finite populations. To answer 
this we have provided illustrative examples from simulations using Kimura's pseudosampling method (Kimura, 1980): Pseudosampling rescales a uniform random variate to have the same variance of that expected under genetic drift as an approximation to the diffusion of alleles in a finite population. The change in an allele's frequency due to deterministic forces, here selection and migration, are then adjusted in each generation. The adjustment is according to the appropriately scaled random variate. This method is a computationally efficient and gives reasonably accurate approximation of genetic drift (Kimura, 1980). Some illustrative results for various configurations in parameter space are given in Fig. D.11. Simulations have been performed with alleles starting at the non-trivial stable equilibrium point (in a deterministic sense) and were jointly iterated each generation until the difference in allele frequencies between the populations collapsed to less than $1 \%$ and the minor allele frequency in each population was less than $1 \%$. We set a maximum upper time bound of $10^{5}$ generations in the simulations, and for plotting purposes have a maximum of $10^{4}$ generations. Because of this bound, an expectation can not be accurately calculated. Instead we show a range of more informative lower percentiles for the time until loss. As modeled here, if the population size is large ( $>100$ diploid individuals) and parameter configurations are far from critical boundaries, a difference in allele frequencies can be maintained for $10^{4}$ generations with a probability greater than $99 \%$. As population sizes decrease, or critical boundaries are approached (with increasing migration, and/or heterozygote fitness, and/or decreasing asymmetric homozygote fitness) the time until loss monotonically decreases.

Note that the evolution of modifying factors such as mate choice discrimination or genetic suppression can be quite rapid in circumstances where there is strong selection (e.g. Soans et al. (1974); McInnis et al. (1996); Charlat et al. (2007)). Because of this we are hesitant to make predictions beyond $10^{4}$ generations.

If the two-deme system is fully symmetrical, $\nu=1$, fixation or loss of the $T$ allele in both populations is equally likely. However, it is found that with even small asymmetries in homozygote fitnesses, $\nu<1$, the probability of fixation of the allele corresponding to the less fit homozygote is dramatically reduced. The proportion of fixation out of the total number that were fixed or lost within the time period considered was $9 \times 10^{-3}$ for $\nu=0.98,8 \times 10^{-4}$ for $\nu=0.97$. No fixations were observed out of $10^{4}$ replicates for $\nu \leq 0.96$. It is likely that genetically modified organisms resulting in underdominance will have reduced fitness relative to wildtypes. This provides a degree of fail-safe into the reversibility of the system; when a local population transformation is disrupted, it is much more likely that the genetic modification will be lost from the wild rather than achieving full fixation. 


\section{References}

Asman, S. M., McDonald, P. T., Prout, T., 1981. Field studies of genetic control systems for mosquitoes. Annu. Rev. Entomol 26, 289-318.

Barton, N. H., 1979. The dynamics of hybrid zones. Heredity 43, 341-359.

Barton, N. H., Rouhani, S., 1991. The probability of fixation of a new karyotype in a continuous population. Evolution 45, 499-517.

Bengtsson, B. O., Bodmer, W. F., 1976. On the increase of chromosome mutations under random mating. Theor. Pop. Biol. 9, 260-281.

Boussy, I. A., 1988. A Drosophila model of improving the fitness of translocations for genetic control. Theor. Appl. Genet. 76, 627-639.

Bürger, R., 2009. Multilocus selection in subdivided populations II. Maintenance of polymorphism under weak or strong migration. J. Math. Biol. 58, 979-997.

Bush, G. L., Case, S. M., Wilson, A. C., Patton, J. L., 1977. Rapid speciation and chromosomal evolution in mammals. Proc. Natl. Acad. Sci. U.S.A. 74, 3942-3946.

Charlat, S., Hornett, E. A., Fullard, J. H., Davies, N., Roderick, G. K., Wedell, N., Hurst, G. D. D., 2007. Extraordinary Flux in Sex Ratio. Science 317, 214.

Curtis, C. F., 1968. Possible use of translocations to fix desirable genes in insect pest populations. Nature 218, 368-369.

Davis, S., Bax, N., Grewe, P., 2001. Engineered underdominance allows efficient and economical introgression of traits into pest populations. J. Theor. Biol. 7, 83-98.

Dye, C., 1984. Models for the population dynamics of the yellow fever mosquito Aedes aegypti. J. Anim. Ecol. 53, 247-268.

Fisher, R. A., 1922. On the dominance ratio. Proc. Roy. Soc. Edinburgh 42, 321-341.

Fisher, R. A., 1937. The wave of advance of advantageous genes. Ann. Eugenics 7, 355-369.

Foster, G. G., Whitten, M. J., Prout, T., Gill, R., 1972. Chromosome rearrangements for the control of insect pests. Science 176, 875-880.

Gould, F., 2008. Broadening the application of evolutionarily based genetic pest management. Evolution 62, 500-510.

Haldane, J. B. S., 1924. A mathematical theory of natural and artificial selection. Part V. Selection and mutation. Proc. Camb. Phil. Soc. 28, 838-844.

Haldane, J. B. S., 1937. The effect of variation in fitness. Am. Nat. 11, 337-349.

Haldane, J. B. S., 1942. Selection against heterozygosis in man. Ann. Eugen 11, 333-340. 
Harewood, L., Schütz, F., Boyle, S., Perry, P., Delorenzi, M., Bickmore, W. A., and Reymond, A., 2010. The effect of translocation-induced nuclear reorganization on gene expression. Genome Res. 20, 554-564.

Hartl, D., Clark, A. G., 1989. Principles of Population Genetics, 2nd Edition. Sinauer Associates, Inc., Sunderland, Massachusetts.

Hedrick, P. W., 1981. The establishment of chromosomal variants. Evolution 35, 322-332.

Hofbauer, J., Sigmund, K., 1998. Evolutionary Games and Population Dynamics. Cambridge University Press, Cambridge.

Ito, J., Ghosh, A., Moreira, L. A., Wimmer, E. A., and Jacobs-Lorena, M. 2002. Transgenic anopheline mosquitoes impaired in transmission of a malaria parasite. Nature 417, 452-455.

Karlin, S., McGregor, J. 1972. Application of method of small parameters to multi-niche population genetic model. Theor. Pop. Biol. 3, 186-209.

Karlin, S., McGregor, J. 1972. Polymorphism for Genetic and Ecological Systems with Weak Coupling. Theor. Pop. Biol. 3, 210-238.

Kimura, M., 1962. On the probability of fixation of mutant genes in a population. Genetics 47, 713-719.

Kimura, M., 1968. Evolutionary Rate at the Molecular Level. Nature 217, 624-626.

Kimura, M., 1980. Average time until fixation of a mutant allele in a finite population under continued mutation pressure: Studies by analytical, numerical and pseudo-sampling methods. Proc. Natl. Acad. Sci. U.S.A. 77, 522-526.

Koochek, M., Harvard, C., Hildebrand, M. J., Allen, M. V., Wingert, H., Mickelson, E., Holden, J. J. A., Rajcan-Separovic, E., Lewis, M. E. S., 2006. 15q duplication associated with autism in a multiplex family with a familial cryptic translocation $\mathrm{t}(14 ; 15)(\mathrm{q} 11.2 ; \mathrm{q} 13.3)$ detected using array-cgh. Clinical Genetics 69, 124-134.

Krafsur, E. S., 1998. Sterile insect technique for suppressing and eradicating insect populations: 55 years and counting. J. Ag. Entomol. 15, 303-317.

Lance, D. R., McInnis, D. O., Rendon, P., Jackson, C. G., 1998. Courtship among sterile and wild Ceratitis capitata (Diptera: Tephritidae) in field cages in Hawaii and Guatemala. Ann. Entomol. Soc. Am. 93 (1179-1185).

Lande, R., 1979. Effective deme sizes during long term evolution estimated from rates of chromosomal rearrangments. Evolution 33, 234-251.

Lande, R., 1985. The fixation of chromosomal rearrangements in a subdivided population with local extinction and colonization. Heredity 54, 323-332.

Laven, H., 1969. Eradicating mosquitoes using translocations. Nature 221, 958-959. 
Lenormand, T., 2002. Gene flow and the limits to natural selection. Trends Ecol. Evol. 17, 183-189.

Li, C. C., 1955. The stability of an equilibrium and the average fitness of a population. Am. Nat. 89, 281-295.

Lorimer, N., Hallinan, E., Rai, K. S., 1972. Translocation homozygotes in the yellow fever mosquito, Aedes aegypti. J. Hered. 63, 158-166.

Magori, K., Gould, F., 2006. Genetically engineered underdominance for manipulation of pest populations: a deterministic model. Genetics 172, 2613-2620.

McInnis, D. O., Lance, D. R., Jackson, C. G., 1996. Behavioral resistance to the sterile insect technique by mediterranean fruit fly (Diptera: Tephritidae) in Hawaii. Ann. Entomol. Soc. Am. 89, 739-744.

Murray, J. D., 2007. Mathematical Biology I: An Introduction, 3rd Edition. Springer.

Nachman, M. W., Searle, J. B., 1995. Why is the house mouse karyotype so variable? Trends Ecol. Evol. 10, 397-402.

Nagylaki, T., Lou, Y., 2001. Patterns of multiallelic polymorphism maintained by migration and selection. Theor. Popul. Biol. 59, 297-313.

Piálek, J., Barton, N. H., 1997. The spread of an advantageous allele across a barrier: The effects of random drift and selection against heterozygotes. Genetics 145, 493-504.

Prout, T., 1978. The joint effects of the release of sterile males and immigration of fertilized females on a density regulated population. Theor. Pop. Biol. 13, 40-71.

Ray, S. M., Park, S. S., Ray, A., 1997. Pollen tube guidance by the female gametophyte. Development 124, 2489-2498.

Rieseberg, L. H., 2001. Chromosomal rearrangements and speciation. Trends Ecol. Evol. 16, 351-358.

Ronce, O., Kirkpatrick, M., 2001. When sources become sinks: migrational meltdown in heterogeneous habitats. Evolution 55, 1520-1531.

Serebrovskii, A. S., 1940. On the possibility of a new method for the control of insect pests. Zoolog. Zhurnal 19, 618-630.

Sinkins, S. P., Gould, F., 2006. Gene drive systems for insect disease vectors. Nat. Rev. Gen. 7, 427-435.

Snell, G. D., 1946. An analysis of translocations in the mouse. Genetics 31, 157-180.

Soans, A. B., Pimentel D., and Soans J. S., 1974. Evolution of Reproductive Isolation in Allopatric and Sympatric Populations. Am. Nat. 108, 117-124. 
Soboleva, T. K., Shorten, P. R., Pleasants, A. B., , Rae, A. L., 2003. Qualitative theory of the spread of a new gene into a resident population. Ecol. Modell. 163, 33-44.

Strogatz, S., 2000. Nonlinear Dynamics and Chaos: With Applications to Physics, Biology, Chemistry, and Engineering (Studies in Nonlinearity). Westview Press. Cambridge, Massachusetts.

Tufto, J., 2001. Effects of releasing maladapted individuals: a demographic-evolutionary model. Am. Nat. 158, 331-340.

Vanderplank, F. L., 1944. Hybridization between glossina species and suggested new method for control of certain species of tsetse. Nature 154, 607-608.

Walsh, J. B., 1982. Rate of accumulation of reproductive isolation by chromosomal rearrangements. Am. Nat. 120, 510-532.

Warner, R. E., 1968. The role of introduced diseases in the extinction of the endemic Hawaiian avifauna. Condor 70: 101-120.

White, M. J. D., 1978. Modes of Speciation. W. H. Freeman and Company. San Francisco, California.

Wiener, A. S., 1942. The Rh factor and racial origins. Science 96, 407-408.

Wright, S., 1931. Evolution in mendelian populations. Genetics 16, 97-159.

Wright, S., 1941. On the probability of fixation of reciprocal translocations. Am. Nat. 75, 513-522. 


\section{Figures}

Fully symmetric

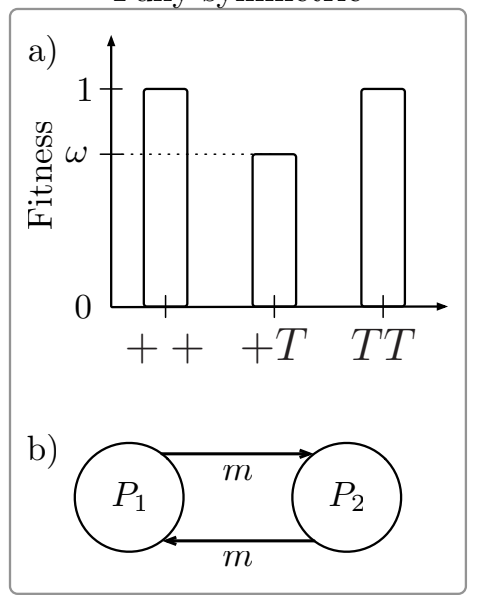

Doubly asymmetric

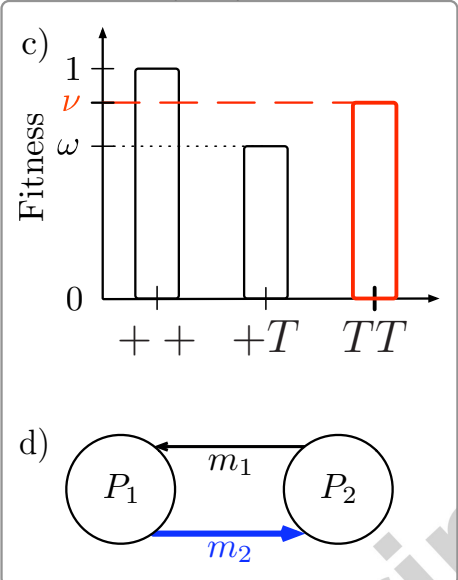

Figure D.1: Left: (a) Genotype configuration of underdominance with the fitness of the homozygotes set to one, $w_{++}=w_{T T}=1$. The reduced fitness of the heterozygote, $w_{T+}=\omega$, is a positive number which is less than one. (b) Migration between the two populations. In each generation, migrants come and go with the rate $m$ $(0 \leq m<0.5)$, such that the fraction of immigrants in each population is $m$. Right: (c) Genotype configuration of underdominance in the asymmetric case. The fitness of the wildtype homozygotes is 1 . The fitness of the transgenic type homozygotes is $\nu \leq 1$. The underdominant fitness of the heterozygotes is $\omega<\nu$. (d) Nonsymmetric migration between the two populations. For each generation, the contributions of immigrants to the next generation in each of the two populations with $p_{1}, p_{2}$ are $m_{1}<0.5$, and $m_{2}<0.5$, respectively. 
a)

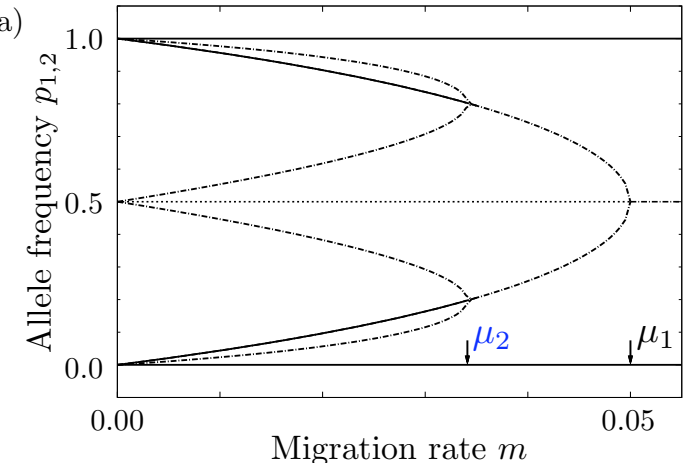

b) $(1,0)$
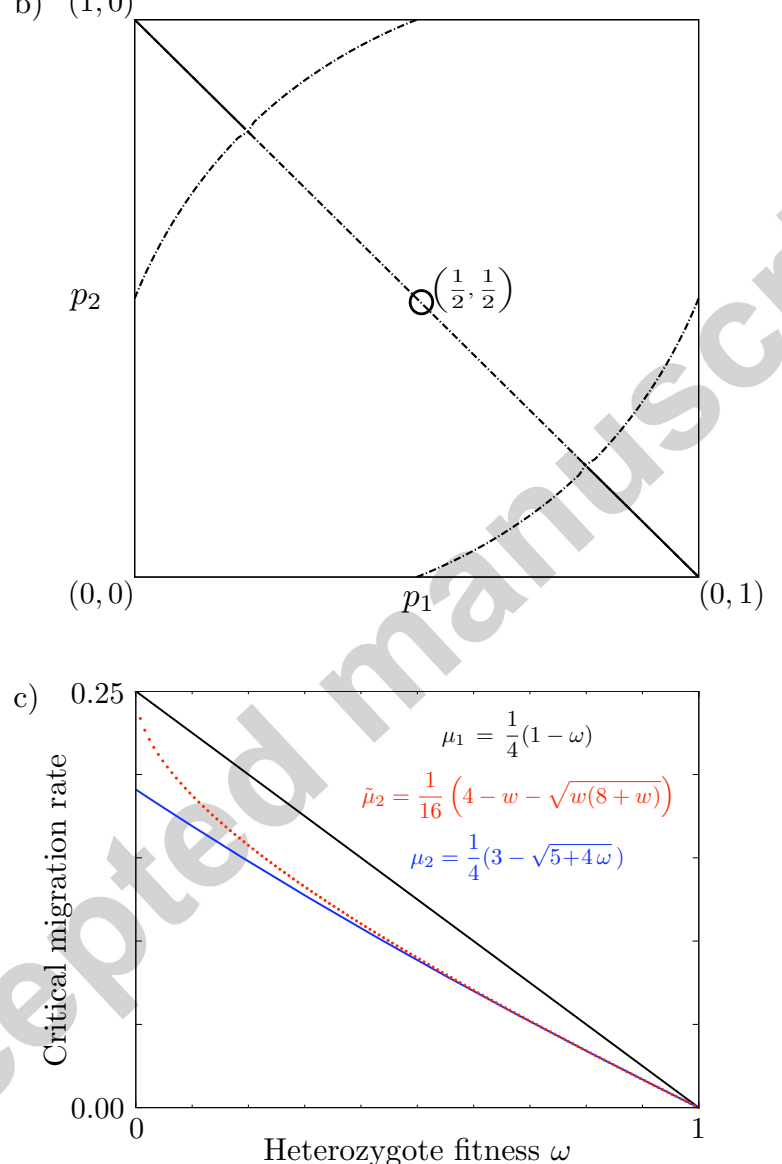

Figure D.2: (a) Projection of the bifurcation diagram showing the allele frequency $p_{1,2}$ in one of the two populations as a function of the control parameter $m$. The positions of the critical points $\mu_{1}(\omega)=0.05$, and $\mu_{2}(\omega) \approx 0.0341$ are indicated by arrows. (b) Projection of the bifurcation diagram to the state space, i.e. the positions of equilibria for any migration rate. Dashed lines and curves describe unstable, dashed-dotted curves describe saddle, and full curves and lines describe stable fixed points in both figures. In both, (a) and (b), the fitness of the heterozygote's is fixed to $\omega=0.8$. Note the difference to Karlin and McGregor (1972a), where a figure of this bifurcation wrongly suggests a different pattern. (c) The two critical points of the fully symmetric system, $\mu_{1}$ (black), and $\mu_{2}$ (shaded), and the approximation of the latter stemming from the linearized system, $\tilde{\mu}_{2}$ (dotted), as a function of the fitness of the heterozygotes $(+T), \omega$. While $\mu_{1}$ is a linear function in $\omega, \mu_{2}$ and $\tilde{\mu}_{2}$ exhibit square root behavior (compare inset and Eqs. (8), (9), and (B.7) in the main text). $\tilde{\mu}_{2}$ approaches $\mu_{2}$ for $\omega$ sufficiently large; The difference vanishes for $\omega \rightarrow 1$. 



$(1,1)$

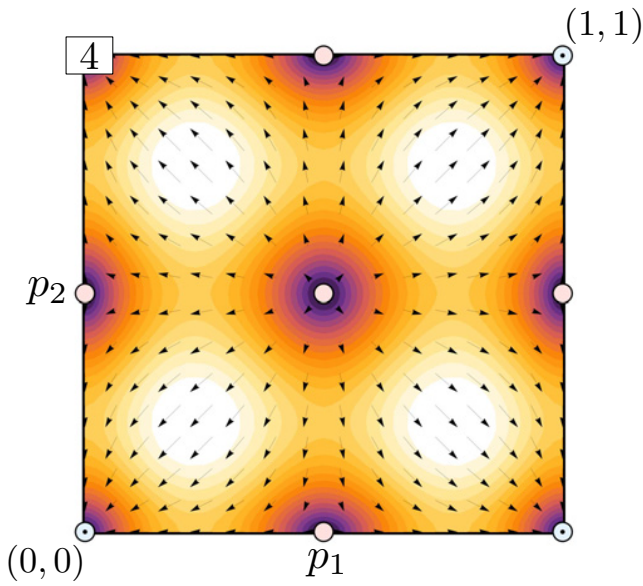

Figure D.3: For the bifurcation pattern with fixed heterozygotes fitness $\omega=0.5$ and critical points $\mu_{1}=0.125$, and $\mu_{2} \approx 0.0886$, four slices (1-4) with different migration rates $m$ are shown. In each of them the state space of the system, $([0,1] \times[0,1])$, is depicted with a phase portrait (arrows) and the absolute rate of change $\sqrt{\left(\Delta p_{1}\right)^{2}+\left(\Delta p_{2}\right)^{2}}$ (shading). The darker the shading the slower the dynamics, the brighter the faster. The equilibrium points are given by disks. Empty disks are unstable (or saddles), disks with a dot are the stable equilibria. (1) For $m=0.13$ there exist only the two trivially stable equilibria at $(0,0)$ and $(1,1)$, as well as the saddle fixed point at the center $(0.5,0.5)$. (2) If migration rate decreases below $\mu_{1}$, for $m=0.1$, we observe two new saddles along the axis $\left(p_{1}, 1-p_{1}\right)$, cf. Eq. (7), and the center becomes fully unstable. (3) For migration below $\mu_{2}, m=0.07$, the former saddles at $\left(x_{1,2}, 1-x_{1,2}\right)$, Eq. (7), become stable and four new equilibria (also saddles) emerge, cf. Figure 1 of Barton and Rouhani (1991). Although they also obey the mirror symmetry, for the four new unstable points an analytical description of their positions is cumbersome; we only locate them via numerical root finding algorithms. (4) For vanishing migration rate the case of two distinct populations with underdominance is recovered. 

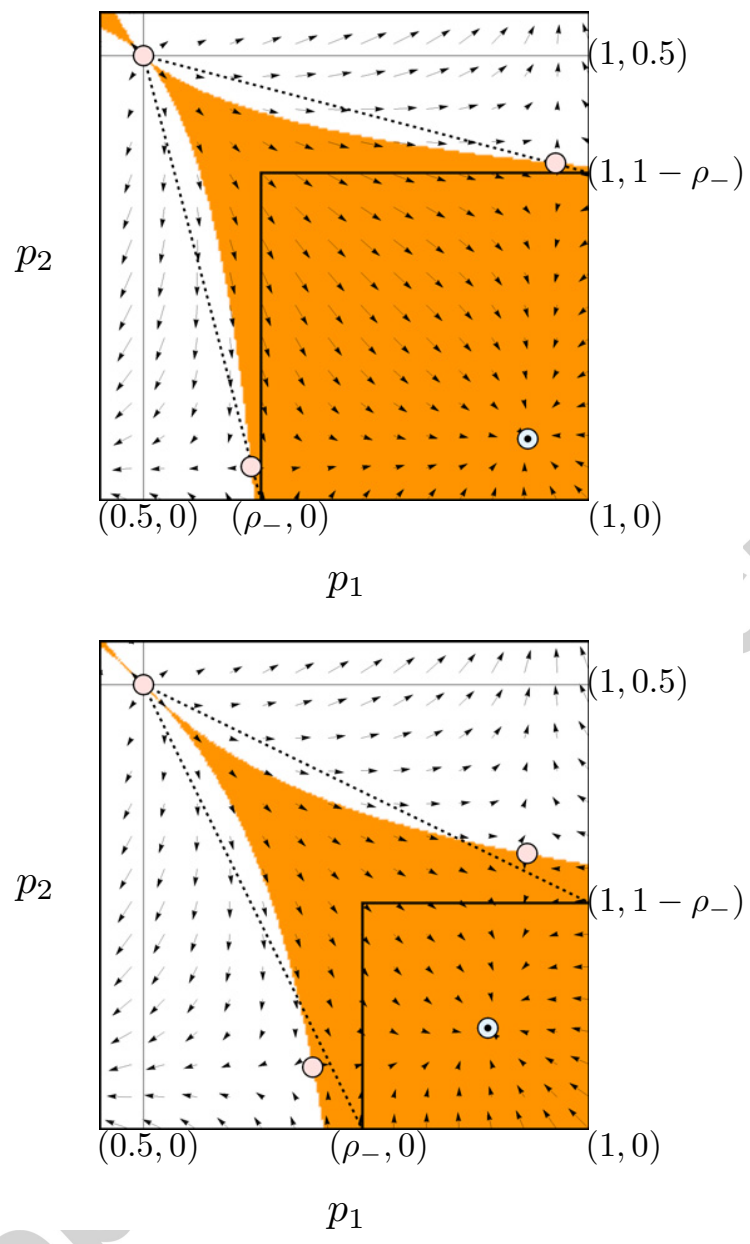

Figure D.4: We show the basin of attraction for heterozygotes fitness of $\omega=0.5\left(\mu_{2}(0.5) \approx 0.0886\right)$ in the lower right quarter of the state space. The migration rates are $m=0.05$ (top), and $m=0.07$ (bottom). The basin in darker shading results from simulations of the dynamic system in discrete time, Eqs. (5) and (6). The thick lines are estimates from the reduction to the one-dimensional flow along the boundaries, cf. Eq. (10): The square in full lines is a conservative underestimate, whereas the quadrilateral limited by the dashed lines and the boundaries serves as an overestimate when $p_{2}$ is not too small and $p_{1}$ is not too high (in natural populations, the migration rate ensures these properties). Although migration is low, it becomes clear that with $m$ increasing both estimates become conservative in a neighborhood of the $\rho_{-}$boundaries. 




Figure D.5: Total mean population fitness of the central unstable equilibrium, $\bar{w}=(1+\omega) / 2$ (full lines), and of the one-dimensional edge approximation for single population release, Eq. (12) (dotted curves), are shown with four different values of heterozygote fitness $\omega$ (values given in the figure). The two estimates are essentially equal except at relatively high migration rates. 

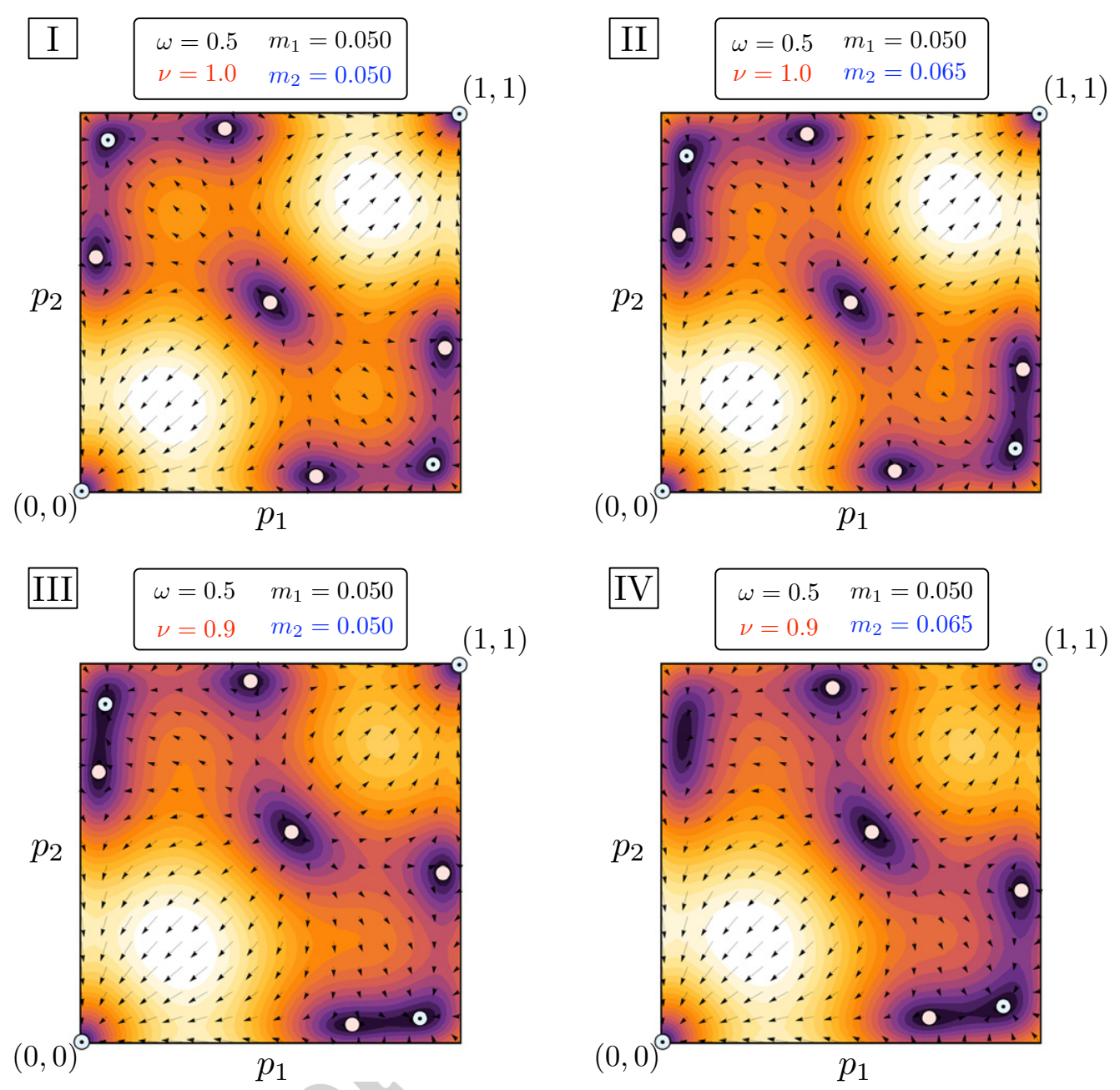

Figure D.6: Symmetry breaking. We show four different scenarios with different degrees of symmetry. $\omega$ denotes the fitness of the heterozygotes, $\nu$ is the fitness of the transgenic homozygous type, which is equal or less than the fitness of the wildtype, with fitness of one. In population $i=1,2$, the frequency of immigrants which contribute to the next generation is $m_{i}$. The exact values of fitnesses and migration rates are given in the boxes above each plot. Disks indicate equilibrium points; empty disks are unstable (or saddle points), disks with a dot are stable equilibria. (I) Maximal symmetry is maintained when the fitness of both homozygotes is equal to one and migration is symmetric, $m_{1}=m_{2}$. In this case there is a point symmetry and one finds two symmetry axes. The important symmetry here is $p_{1} \rightarrow 1-p_{1}$. (II) When migration is asymmetric both symmetry axes are lost, but there is still a point symmetry in the center $(0.5,0.5)$. (III) For symmetric migration but lower fitness of the transgenic homozygotes, $\omega<\nu<1$, the point symmetry and the important symmetry axis are lost. The unstable equilibrium in the central area is shifted along the axis $\left(p_{1}, p_{1}\right)$. (IV) For unequal migration rates and unequal homozygotes fitness all symmetry is lost, but the central unstable equilibrium remains at the axis $\left(p_{1}, p_{1}\right)$. In this case, only one nontrivial fixed point is stable. 


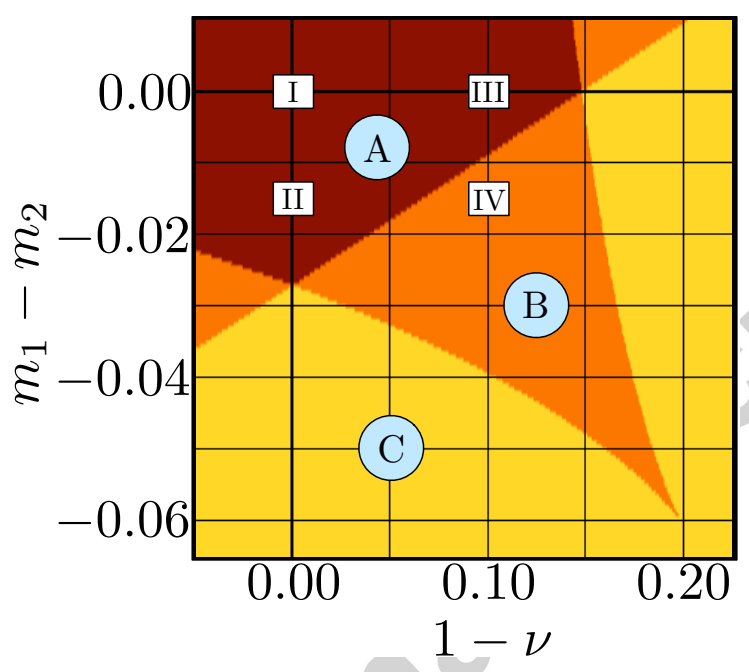

Figure D.7: Phase diagram of a system of two populations with an underdominant allele coupled by two migration rates. We show the difference of migration rates, $m_{1}-m_{2}$, on the ordinate and the difference in homozygote fitnesses, $w_{++}-w_{T T}=1-\nu$, on the abscissa. For each pair of these differences, we evaluate whether the system reaches an internal (non-trivial) stable fixed point, given it has been initiated inside the basin of attraction (compare Fig. D.4). The system has been simulated using Eqs. (16), (17). We can identify three different phases: (A) The system has two internal stable fixed points (darkest shading). (B) The system has only one internal stable fixed point (intermediate shading). (C) The system does not have any internal stable fixed point (light shading). We also link back to Fig. D.6 and locate the plots in this phase diagram: (I) Full symmetry is maintained, cf. Fig. D.3. (II) The system is moved along the axis of equal homozygote fitness, $1-\nu=0$. (III) The system is moved along the axis of equal rates of migration, $m_{1}-m_{2}=0$. (IV) No symmetry is maintained and we find only one internal stable fixed point. 


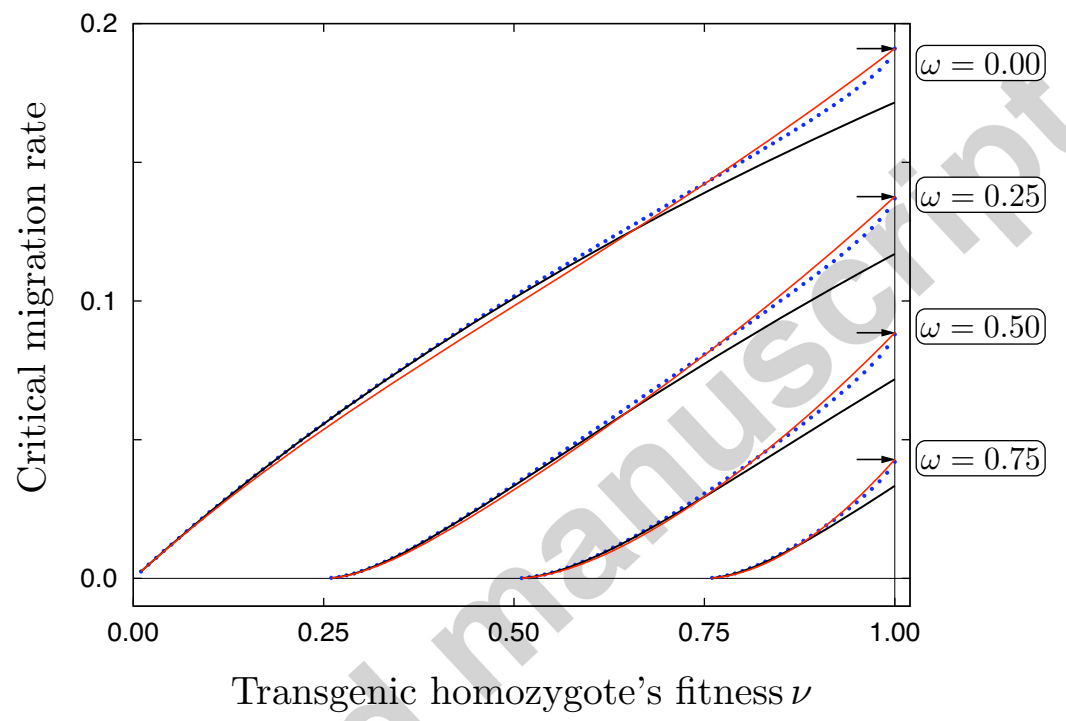

Figure D.8: Critical migration rates with unequal homozygote fitnesses for a range of illustrative heterozygote fitness values, $\omega$. The right edge of the plot, $\nu=1$, corresponds to $\mu_{2}$ in the fully symmetric model $(\nu=1$, arrows). As the $T T$ homozygote fitness declines relative to the wildtype, ++ , the critical migration rate also declines as the system is destabilized. This has been determined numerically (dots) and the simulated result approaches the approximation analytically, derived from the edge of the system for smaller $\nu$ (black). A simple non-linear weighted average approximates critical values of $m$ across the range of $\nu$ and $\omega$ (shaded), compare Eq. (20). 

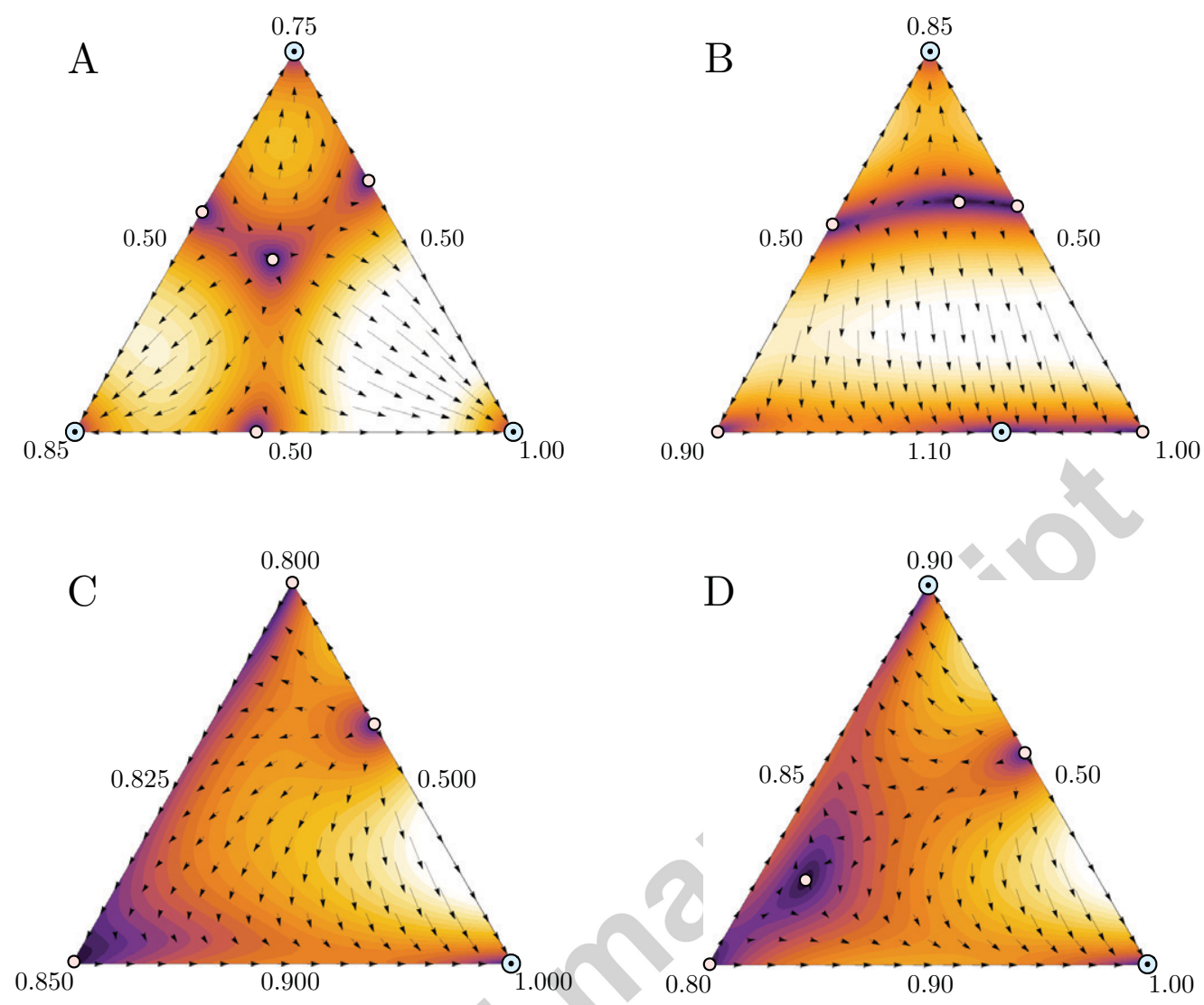

Figure D.9: Dynamics of a three allele model in a single population. The shading indicates rate of change in joint allele frequencies on a scale from dark (slow) to light (fast). Arrows indicate direction of change. Unstable equilibria as well as saddles are indicated with an empty disk, stable fixed points are black dotted disks. At the vertexes of the simplex the population is fixed for one of the three alleles, and the corresponding allele is lost from the population at points along the opposing edge. In all four panels the representation is wildtype + in the lower right corner, type $R$ in the lower left corner, and type $T$ in the top corner. The genotypic fitness values are given in each panel: near the vertices the homozygote fitnesses $\left(\nu_{++}=1, \nu_{R R}, \nu_{T T}\right)$, along the edges the heterozygote fitnesses $\left(\omega_{+R}, \omega_{R T}, \omega_{+T}\right)$. (A) Full three-way underdominance. $\omega_{+R}=\omega_{R T}=\omega_{+T}=0.5$, and $\nu_{++}=1, \nu_{R R}=0.85, \nu_{T T}=0.75$. With fitness asymmetry among the homogenous genotypes, the central unstable point moves towards higher frequencies of the less fit genotypes. (B) Overdominance between two wildtype alleles. $\omega_{+R}=1.1, \omega_{R T}=\omega_{+T}=0.5$, and $\nu_{++}=1, \nu_{R R}=0.9, \nu_{T T}=0.85$. Overdominance between the remaining alleles raises the transformation threshold of the modified allele. (C) $\omega_{+R}=0.9, \omega_{R T}=$ $0.825, \omega_{+T}=0.5$, and $\nu_{++}=1, \nu_{R R}=0.85, \nu_{T T}=0.8$. If directional selection with $R$ exists with fitness increasing away from a less fit $T T$ homozygote, fixation of $T$ is unstable despite heterozygote disadvantage (underdominance) with wildtype. This may provide insight into how some types of underdominant changes can evolve between species, by transiting via intermediate alleles. (D) $\omega_{+R}=0.9, \omega_{R T}=0.85, \omega_{+T}=0.5$, and $\nu_{++}=1, \nu_{R R}=0.8, \nu_{T T}=0.9$. If directional selection exists and $R R$ is less fit than the other homozygotes, underdominance can remain bi-stable between $T T$ and wildtype ++ . 


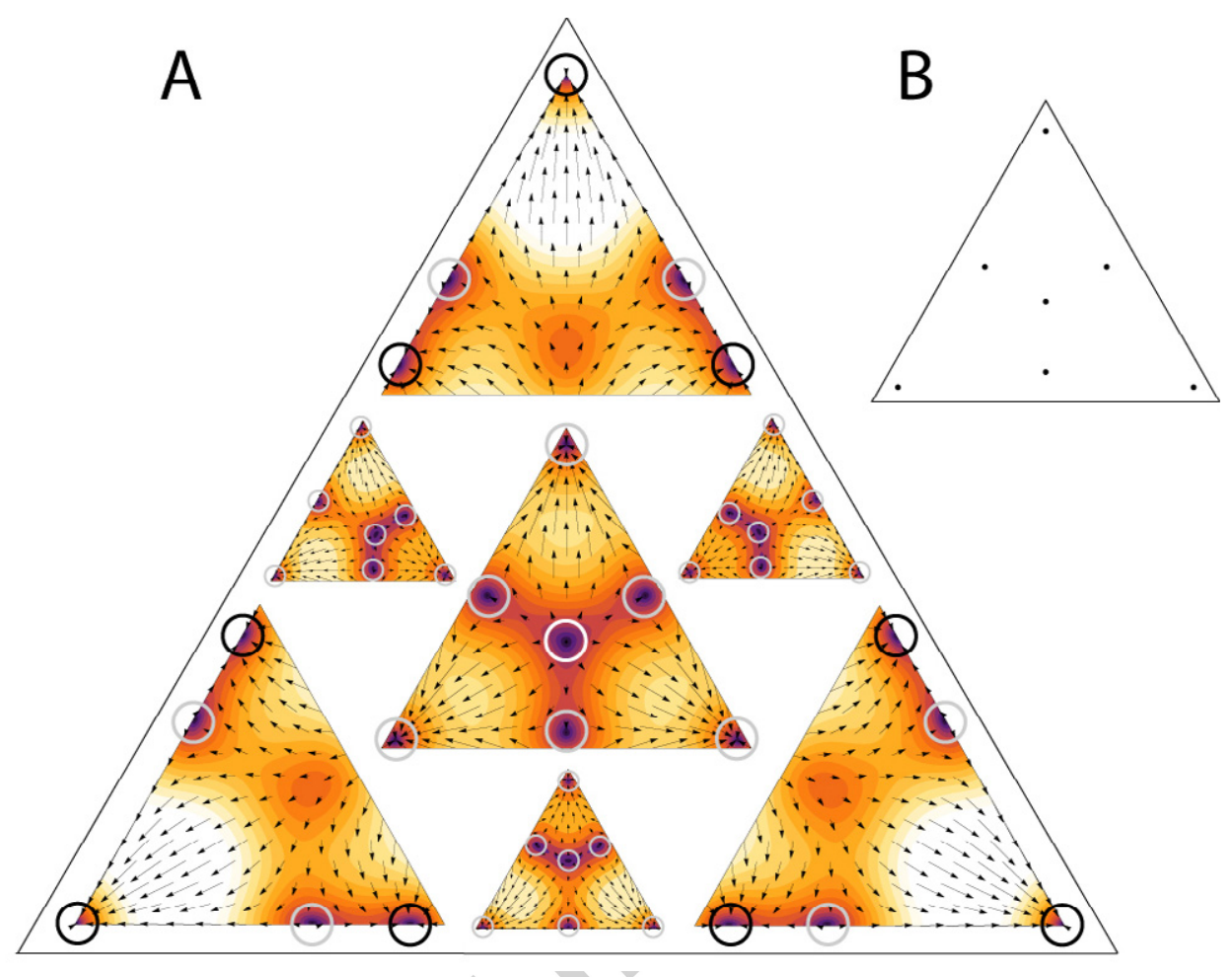

Figure D.10: A schematic illustrating the fixed points and their stability in slices of the four-dimensional state space of three alleles in two populations. (A) A useful visualization in this context is a two-dimensional simplex for population $1\left(\mathcal{S}_{1}^{2}\right)$, and at each point within this simplex an additional two-dimensional simplex also exists for population $2\left(\mathcal{S}_{2}^{2}\right.$, four total dimensions). Consider the large outlined triangle to be $\mathcal{S}_{1}^{2}$, within this illustrative $\mathcal{S}_{2}^{2}$ s are arranged according to their relative positions within $\mathcal{S}_{1}^{2}$. In this example, all homozygotes have equal fitnesses, all heterozygotes have half the fitness of the homozygotes (cf. D.9A), and the migration rate is $m=0.06$. The approximate positions of fixed points are indicated with open circles, which may not exist in precisely these illustrative slices, but are expected to be nearby in the four-dimensional space. The central unstable equilibrium is outlined in white, saddle points in gray, and stable equilibria in black. Note that some points that appear to be stable equilibria in two dimensions are actually saddle points in four dimensions. At this migration rate six of the 49 possible fixed points have merged and disappeared, so a total of 43 fixed points are indicated. Of interest are the non-trivial stable fixed points on the outside edges of the "corner" $\mathcal{S}_{2}^{2} \mathrm{~s}$, where the allele is at a high frequency in one population and at low frequency in the alternate population. (B) The actual positions of the internal simplexes in panel $\mathbf{A}$ are indicated as points within the first population simplex. Except for the central point, these are closer to the edges then can be shown in $\mathbf{A}$. 



Figure D.11: Time until loss of underdominant polymorphism in populations of finite size. Results from 10,000 simulated replicates are plotted. The 50th percentile is represented by dark circles, the lower 5th percentile by medium shaded squares, and the 1st percentile by light triangles (color in the on-line version). Unless otherwise indicated, all points are plotted with $\omega=0.5, \nu=1, m=0.05$, and the diploid population size $N=100$. All times are in generations. (A) The destabilizing effect of small population sizes is illustrated. (B) The destabilizing effect of unequal homozygote fitnesses. (C) The effect of increasing heterozygote fitness. (D) The effect of increasing migration rates. 